Mathematisches Forschungsinstitut Oberwolfach

Report No. 49/2011

DOI: $10.4171 /$ OWR/2011/49

\title{
Arbeitsgemeinschaft: Quantum Ergodicity
}

\author{
Organised by \\ Ulrich Bunke, Regensburg \\ Stephane Nonnenmacher, Gif-sur-Yvette \\ Roman Schubert, Bristol \\ October 8th - October 14th, 2011
}

\begin{abstract}
Quantum Ergodicity aims at understanding the eigenstates of quantum mechanical systems admitting chaotic classical limiting dynamics. A paradigmatic system is the Laplace-Beltrami operator on a compact manifold of negative sectional curvature: its classical limit is the geodesic flow on the manifold, which is of Anosov type. Although no explicit expression is available for the eigenstates, one may use various tools from semiclassical analysis in order to gather some partial information on their structure. The central result (Quantum Ergodicity Theorem) states that almost all eigenstates are equidistributed over the energy shell, in the semiclassical limit, provided the classical system is ergodic. The lectures review the background techniques of semiclassical analysis and ergodic theory, give several versions of the QE theorem, and present several extensions of the result, which apply to specific systems, for instance chaotic systems enjoying arithmetic symmetries.
\end{abstract}

Mathematics Subject Classification (2000): 35P20, 81Q50.

\section{Introduction by the Organisers}

Quantum ergodicity is a subfield of quantum chaos, which can itself be considered a subfield of semiclassical analysis: the latter aims at describing quantum systems in the semiclassical limit. Originally, a quantum system is defined by a Schrödinger operator (the quantum Hamiltonian) $P(\hbar)$ acting on $L^{2}\left(\mathbb{R}^{3}\right)$, but this definition can be generalized in various ways, for instance, by considering Schrödinger, or Laplace-Beltrami operators on a Riemannian manifold $(X, g)$. This Hamiltonian depends on Planck's constant $\hbar>0$, which is assumed very small: mathematically, the semiclassical limit consists in studying the asymptotical regime $\hbar \downarrow 0$. In this limit, quantum mechanics "converges to" classical mechanics, which is described 
by a Hamiltonian dynamical system on the phase space $T^{*} \mathbb{R}^{3}$ (more generally $T^{*} X$ ). Yet this "convergence" is rather singular, which makes semiclassical analysis interesting and nontrivial. Some tools of semiclassical analysis, which describe the nature of this "convergence", were explained in the first two lectures.

The time evolution is governed by the spectrum of $P(\hbar)$ (in case of discrete spectrum, the eigenvalues and eigenstates).

Because we are interested in the spectral properties of the quantum system, we first need a good understanding of the stationary, or long time, properties of the classical Hamiltonian dynamical system. This information is provided by ergodic theory, which describes the invariant probability measures on phase space. Somewhat paradoxically, ergodic theory is best understood for two antipodal types of Hamiltonian systems. On one side, the fully (Liouville-)integrable systems, for which one can (at least locally) construct a full set of action-angle coordinates for which the dynamics is simple (quasiperiodic). At the other extreme, the (fully) chaotic systems, where the only invariant of the motion is the energy, all trajectories are unstable, and the dynamics mixes up the whole energy shell. General ergodic theory tools, as well as specific results in the case of chaotic Hamiltonian systems, were presented in the lectures by I.Khayutin, D.Schleicher and R.Gunesch.

The aim of quantum chaos is to specifically study the Schrödinger operators for which the limiting classical mechanics is chaotic. We have focussed on systems for which the individual energy shells are compact, resulting in discrete spectra $\left(E_{\hbar, n}\right)$ for the Schrödinger operators. One paradigmatic system is the Laplace-Beltrami operator on a compact Riemannian manifold of negative sectional curvature (for which the limiting classical flow, namely the geodesic flow, is uniformly hyperbolic, the strongest possible form of chaos), or on certain billiards (like the stadium billiard on $\mathbb{R}^{2}$ ) such that the broken geodesic flow is chaotic. In this case, the semiclassical limit $\hbar \rightarrow 0$ is equivalent with the high frequency limit $\lambda \rightarrow \infty$, where $\lambda^{2}$ is the eigenvalue of the Laplacian.

One aim of quantum chaos is to describe the eigenvalues $\left(E_{\hbar, n}\right)$ and corresponding eigenfunctions $u_{\hbar, n} \in L^{2}$ of $P(\hbar)$, in the limit $\hbar \rightarrow 0$. In such chaotic systems it is impossible to separate variables, neither at the classical nor quantum levels. Hence, these spectral datas do not admit any explicit, or even approximate representation. Still, by using the connection with classical mechanics, semiclassical analysis is able to collect some nontrivial, yet rather "rough" information on this spectrum.

"Quantum Ergodicity", as a research topic, focusses on the description of the eigenfunctions $u_{\hbar, n}$ of such systems. These functions strongly oscillate on a spatial scale $\sim \hbar$, so they become singular in the limit $\hbar \rightarrow 0$. Nevertheless, the macroscopic structure of these eigenfunctions lends itself to some analysis. Since $\left|u_{\hbar, n}(x)\right|^{2}$ represents the probability density of the quantum particle at the point $x$, it makes sense to consider the integral of this probability over a fixed domain $D \subset X$, which represents the probability to find the particle (in the stationary state $u_{\hbar, n}$ ) to be observed in this domain. One can jointly study localization in 
space and momentum variables using phase space quantum representations: as explained in C.Neira Jimenez's lecture, it makes sense to measure the probability of presence $P_{\hbar, n}(\Omega)$ of the particle in a (macroscopic) phase space domain $\Omega \subset T^{*} X$. This probability does not depend much on the fluctuations of the density at the quantum scale, but rather on its macroscopic fluctuations. One can then study the semiclassical limits of the probabilities $P_{\hbar, n}(\cdot)$, which represent the asymptotic macroscopic phase space distribution of the eigenmodes. These limit distributions are called semiclassical measures, they are localized on single energy shells, and are necessarily invariant under the classical dynamics. The main question of "Quantum Ergodicity", as a research topic, is:

What are the possible semiclassical measures? Do they encompass all possible classically invariant measures?

If the limiting classical system is chaotic (in particular, if it is ergodic with respect to the Liouville measure), this question can be partially answered by a quantum ergodicity theorem. This theorem states that for almost every eigenfunction $u_{\hbar, n}$, the probability $P_{\hbar, n}(\Omega)$ is asymptotically given by the Liouville measure of $\Omega$; thanks to the ergodicity assumption, this is also the asymptotic fraction of time almost every initial phase space point will visit $\Omega$ in the course of the classical evolution. One says that these eigenfunctions $u_{\hbar, n}$ are macroscopically equidistributed on the energy shell.

This QE theorem is robust: its proofs are rather elementary, once one has at its disposal a few basic semiclassical properties. As a result, QE holds for a wide variety of systems: the initial proof was given for chaotic geodesic flows, but it extends to more general chaotic smooth Hamiltonian flows (I.Witt's lecture). It can be further extended to toy models like quantized chaotic maps (K.Fritzsch's lecture) or certain quantum graphs (P.Gmeiner's lecture). Its extension to vectorvalued systems leads to interesting questions on the intertwining between internal and external degrees of freedom, and some specific geometric flows, like the frame flow (S.Goette's lecture), or flows on higher rank locally symmetric spaces (M.Olbrich's lecture). QE can also be proved for systems with singularities like chaotic billiards (N.Schippkus's lecture), as long as these singularities occupy a part of phase space of Liouville measure zero. The converse of the QE theorem is also interesting: QE alone does not guarantee that the limiting classical system is ergodic (H.Mizutani's lecture).

The QE theorem naturally leads to various questions. For instance, what is the rate of convergence to the Liouville measure? (H.Then's lecture) Also, does there exist exceptions to the macroscopic distribution of eigenstates? In the textbook case of a compact manifold of negative sectional curvature, there exits many points (e.g. periodic points) with asymptotical behaviour different from the Liouville measure (equivalently, there exist many different classical invariant probability measures). Can certain exceptional eigenmodes of the quantum system behave like some of these invariant measures? The negative answer to this question is 
called the quantum unique ergodicity property (QUE). It states that quantum mechanics selects the equidistribution as the only possible macroscopic behaviour of eigenstates. It has been conjectured to hold for the Laplace-Beltrami operator on manifolds of negative curvature. So far it has been proven only for very specific manifolds, namely surfaces of constant curvature enjoying a rich arithmetic structure, embodied by a commutative algebra of Hecke operators commuting with the Laplacian. It is then natural to consider only joint eigenbases of these commuting operators; these modes were proved to be all asymptotically equidistributed, a property sometimes denoted as Arithmetic QUE (the lectures by L.Rosenzweig, N.Raulf and B.Winn treat 3 different systems admitting such arithemtic structures).

On the opposite, without these arithmetic symmetries, the possibility of exceptional eigenmodes remains open. Numerical computations of Laplacian eigenmodes on 2D chaotic billiards have shown the possibility of strong enhancements of the probability density in the neighbourhood of certain periodic orbits. It remains unclear whether these enhancements (scars) persist in the high frequency limit, and if they are strong enough to modify the macroscopic distribution (K.Kröncke's lecture). In particular, the possible existence of strong scars, that is families of eigenstates asymptotically concentrating (in the $L^{2}$ sense) along one or several periodic orbits, remained open until recently.

Results on this question were first obtained in the framework of certain quantum chaotic maps, like the quantum "cat" maps (hyperbolic automorphisms of the $2 \mathrm{D}$ torus), for which the algebraic structure allows some explicit computations. On the one hand, these maps are equipped with arithmetic symmetries ("Hecke" operators), so one can also restrict oneself to joint eigenstates. The latter were shown to be all asymptotically equidistributed (B.Winn's lecture). On the other hand, due to the possibility of very large spectral degeneracies, one can construct sequences of exceptional eigenstates, with half of the probability concentrated along some periodic orbit, the other half being equidistributed on the torus. Such constructions are very specific to these linear automorphisms, but they show that the QUE conjecture does not hold for all quantized hyperbolic systems (H.Ueberschär's lecture)

A counterexample to QUE was also obtained for the (much more physical) stadium billiard: numerics had observed eigenstates strongly concentrating along the 1D family of bouncing ball orbits, which are not hyperbolic. It was recently proved that, indeed, some eigenstates must (at least partially) concentrate along these orbits, thereby disproving QUE for such billiards (D.Damanik's lecture).

A recent approach has been developed in the case of hyperbolic chaotic systems, to show that not all invariant measures can be obtained as semiclassical measures; in particular, eigenstates cannot fully concentrate near periodic orbits. The argument is based on hyperbolic dispersion estimates, which reflect both the minimal delocalization due to Heisenberg's uncertainty principle, and the classical hyperbolicity. The second ingredient is the entropy of an invariant measure, which measures its complexity (in the information theoretic sense) but also gives 
information on its localization. Putting the two ingredients together one obtains nontrivial lower bounds on the entropy of a semiclassical measure, which, roughly speaking, show that the semiclassical measure must be at least half delocalized (S.Dyatlov's lecture).

Although most of the participants were originally not familiar with the topic and the accompanying methods, the talks they gave showed that they had spent a large amount of time and effort preparing their lectures. This effort was especially remarkable in the case of the 5 graduate students and 4 postdocs presenting talks. We also realized that the material we had including in the lecture proposal was, most of the time, much too heavy for a 1 hour presentation. Nevertheless, the speakers managed to operate an intelligent selection among this material, such as to present reasonable self-contained talks. 



\section{Arbeitsgemeinschaft: Quantum Ergodicity}

\section{Table of Contents}

Bernd Ammann h-pseudodifferential calculus on $\mathbb{R}^{d}$ and on compact manifolds, Egorov's

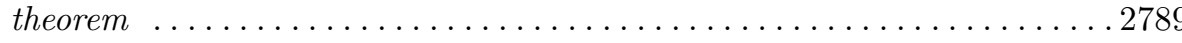

Carolina Neira Jimenez

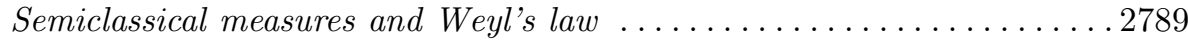

Ilya Khayutin

Concepts from Ergodic Theory .............................22791

Dierk Schleicher

Hyperbolic Dynamics ..................................22795

Ingo Witt

Quantum ergodicity for smooth Hamiltonian flow ................. 2800

Nils Schippkus

Quantum Ergodicity for Ergodic Billiards ..................... 2804

Karsten Fritzsch

Quantum Ergodicity for Quantum Maps on the Torus .............2805

Haruya Mizutani

Reverse quantum ergodicity ..............................2 2808

Peter Gmeiner

Quantum ergodicity for quantum graphs $\ldots \ldots \ldots \ldots \ldots \ldots \ldots \ldots \ldots \ldots$

Sebastian Goette

Quantum Ergodicity for Systems ..........................2811

Holger Then

The rate of quantum ergodicity ............................. 2813

Lior Rosenzweig

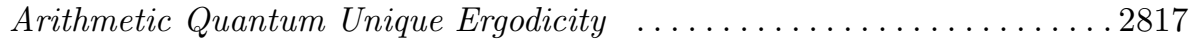

Nicole Raulf

QUE for the modular surface (after Luo and Sarnak) .............2818

Brian Winn

Quantum unique ergodicity for the quantised toral automorphisms ....2820

Klaus Kröncke

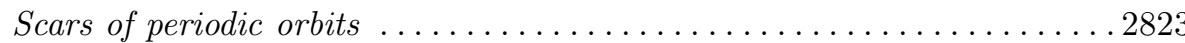


Henrik Ueberschär

A Counterexample to QUE

David Damanik

Hassell's Proof of Quantum Non-Unique Ergodicity for the Bunimovich

Stadium ........................................... 2828

Semyon Dyatlov

Entropy bounds for semiclassical measures

2831 


\section{Abstracts \\ $h$-pseudodifferential calculus on $\mathbb{R}^{d}$ and on compact manifolds, Egorov's theorem \\ BERND AMMANN}

We develop the $h$-pseudodifferential calculus, following the lecture notes by Evans and Zworski [1]. We explain standard and Weyl quantization. A product on symbols is defined which corresponds to the composition of operators after Weyl quantization, and formulas for the calculation of this product are presented. We sketch how to adapt these definitions to compact manifolds, and briefly discuss the dependence on the choice of coordinates and cut-off functions. At the end of the talk we prove Egorov's theorem.

\section{REFERENCES}

[1] L.C. Evans, M. Zworski. Lectures on semiclassical analysis. http://math.berkeley.edu/ zworski/

\section{Semiclassical measures and Weyl's law Carolina Neira Jimenez}

This talk is divided in two parts: In the first part, we give the basic properties of semiclassical defect measures, and in the second one, we develop the functional calculus for pseudodifferential operators and we use it to prove the local and integrated versions of Weyl's law.

\section{Semiclassical measures}

Here we follow Sections 5.1 and 5.2 of [2].

Consider a collection of functions $\{u(h)\}_{0<h \leq h_{0}}$ that is bounded in $L^{2}\left(\mathbb{R}^{n}\right)$. Suppose

$$
a \in S=\left\{a \in C^{\infty}\left(\mathbb{R}^{2 n}\right): \forall \alpha \in \mathbb{N}^{2 n} \exists C_{\alpha}:\left|\partial^{\alpha} a\right| \leq C_{\alpha}\right\} .
$$

Then the Weyl quantization of $a, a^{w}$, satisfies the estimate

$$
\left\|a^{w}(x, h D)\right\|_{L^{2} \rightarrow L^{2}} \leq C \sup _{\mathbb{R}^{2 n}}|a|+O\left(h^{1 / 2}\right) \text { as } h \rightarrow 0 .
$$

Using this estimation for $a \in C_{c}^{\infty}\left(\mathbb{R}^{2 n}\right)$ and the Riesz Representation Theorem, we prove the following

Theorem 1. There exists a Radon measure $\mu$ on $\mathbb{R}^{2 n}$ and a sequence $h_{j} \rightarrow 0$ such that, for any symbol $a \in S$,

$$
\left\langle a^{w}\left(x, h_{j} D\right) u\left(h_{j}\right), u\left(h_{j}\right)\right\rangle \rightarrow \int_{\mathbb{R}^{2 n}} a(x, \xi) d \mu .
$$

The measure $\mu$ is real and nonnegative. 
Definition 1. We call $\mu$ a microlocal defect measure (quantum limit) associated with the family $\{u(h)\}_{0<h \leq h_{0}}$.

Given a real elliptic symbol $p$, consider the operator $P(h)=p^{w}(x, h D)$. Suppose that $\{u(h)\}_{0<h \leq h_{0}}$ is such that $\|u(h)\|_{L^{2}}=1$ and let $\mu$ be a microlocal defect measure associated to this family. Then

(1) If $\|P(h) u(h)\|_{L^{2}}=o(1)$ as $h \rightarrow 0$, then $\operatorname{supp} \mu \subset p^{-1}(0)$.

(2) If $\|P(h) u(h)\|_{L^{2}}=o(h)$ as $h \rightarrow 0$, then $\int_{\mathbb{R}^{2 n}}\{p, a\} d \mu=0$ for every $a \in$ $C_{c}^{\infty}\left(\mathbb{R}^{2 n}\right)$.

\section{WEYL'S LAW}

Here we follow Chapter 8 of [1] and Sections 6, 9 and Appendix E of [2].

Given a smooth function (potential) $V: \mathbb{R}^{n} \rightarrow \mathbb{R}$ growing to infinity as $|x| \rightarrow \infty$, the symbol $p(x, \xi)=|\xi|^{2}+V(x)$ provides interesting information about the corresponding operator $P(h):=P(x, h D)=-h^{2} \Delta+V$, where $\Delta$ is the Laplacian. We focus upon learning how $p$ controls the asymptotic distribution of the eigenvalues of $P(h)$ in the semiclassical limit $h \rightarrow 0$. The spectrum of $P(h)$ is discrete and we have a complete orthonormal set of eigenfunctions $u_{j}(h)$ in $L^{2}\left(\mathbb{R}^{n}\right)$ :

$$
P(h) u_{j}(h)=E_{j}(h) u_{j}(h) .
$$

Theorem 2 (Weyl's law). For each $a<b$, we have

$$
\#\left\{j: a \leq E_{j}(h) \leq b\right\}=\frac{1}{(2 \pi h)^{n}}\left(\operatorname{Vol}_{T^{*} \mathbb{R}^{n}}\left\{a \leq|\xi|^{2}+V(x) \leq b\right\}\right) \text { as } h \rightarrow 0 .
$$

Let $M$ be a compact Riemannian manifold, and let $\Delta$ be the Laplace-Beltrami operator on $M$. Given a potential $V \in C^{\infty}(M)$, we define the Schrödinger operator

$$
P(h):=-h^{2} \Delta+V(x) .
$$

From Riesz Theorem on the discreteness of the spectrum of a compact operator, we conclude that the spectrum of $(P+i)^{-1}$ is discrete, with an accumulation point at 0 . Choosing $\left\{u_{j}(h)\right\}$ as in (1) we can write

$$
P(h)=\sum_{j=1}^{\infty} E_{j}(h) u_{j}(h) \otimes \overline{u_{j}(h)} .
$$

This spectral decomposition gives a functional calculus:

$$
f(P(h))=\sum_{j=1}^{\infty} f\left(E_{j}\right)(h) u_{j}(h) \otimes \overline{u_{j}(h)} .
$$

For $f \in \mathcal{S}(\mathbb{R})$ we investigate the pseudodifferential character of $f(P)$, by using a different expression for $f(P)$.

Definition 2. A function $\tilde{f} \in \mathcal{S}(\mathbb{C})$ is an almost analytic extension of $f$ if $\tilde{f}_{\mid \mathbb{R}}=f$, $\overline{\partial_{z}} \tilde{f}(z)=O\left(|\Im z|^{\infty}\right)$ and $\operatorname{supp} \tilde{f} \subset\{z:|\Im z| \leq 1\}$. 
Let us denote by $d m(z)$ the Lebesgue measure on $\mathbb{C}$. Then Helffer-Sjöstrand formula gives a functional calculus for pseudodifferential operators:

$$
f(P(h))=\frac{1}{\pi i} \int_{\mathbb{C}} \overline{\partial_{z}} \tilde{f}(z)(P(h)-z)^{-1} d m(z) .
$$

The operator $f(P(h))$ belongs to the space $\Psi^{-\infty}(M)$ and is a trace-class operator with symbol

$$
\sigma(f(P(h)))(x, \xi)=f\left(|\xi|^{2}+V(x)\right) .
$$

With this at hand it is possible to prove the following

Theorem 3 (Weyl's law for compact manifolds). For each $a<b$, we have as $h \rightarrow 0$

$$
\#\left\{j: a \leq E_{j}(h) \leq b\right\}=\frac{1}{(2 \pi h)^{n}}\left(\operatorname{Vol}_{T^{*} M}\left\{a \leq|\xi|^{2}+V(x) \leq b\right\}+o(1)\right) .
$$

Theorem 4 (Weyl's Theorem generalized). Let $B \in \Psi^{0}(M)$. Then we have as $h \rightarrow 0$

$$
(2 \pi h)^{n} \sum_{a \leq E_{j} \leq b}\left\langle B u_{j}, u_{j}\right\rangle \longrightarrow \iint_{p^{-1}([a, b])} \sigma(B) d x d \xi .
$$

\section{REFERENCES}

[1] M. Dimassi, J. Sjöstrand, Spectral asymptotics in the semiclassical limit. LMS Lecture Note Series 268, Cambridge University Press, 1999.

[2] L.C. Evans, M. Zworski. Lectures on semiclassical analysis. http://math.berkeley.edu/ zworski/

\section{Concepts from Ergodic Theory ILYA KHAYUTIN}

This talk presents basic notions from ergodic theory with some treatment of the subject of entropy. The focus is on results and proofs are omitted.

\section{Ergodic Theorems}

Our primary objects of study are dynamical systems which preserve a probability measure.

Definition 1. Given a probability space $(X, \mathcal{B}, \mu)$, a measurable transformation $T: X \rightarrow X$ is measure preserving (MPT) if $\forall A \in \mathcal{B}: \mu\left(T^{-1}(A)\right)=\mu(A)$.

A measurable $\mathbb{R}$-action $\phi_{t}$ is measure preserving if $\forall A \in \mathcal{B} \forall t \in \mathbb{R}: \mu\left(\phi_{t}{ }^{-1}(A)\right)=$ $\mu(A)$.

The onset of ergodic theory is in problems regarding the relationship between orbit averages of a function and its measure integral. The first deep theorem in this area is the pointwise ergodic theorem. 
Pointwise Ergodic Theorem (PET) (Birkhoff). Let $T: X \rightarrow X$ be a MPT w.r.t. $\mu$ and $f \in L_{\mu}^{1}(X)$ then:

$$
\lim _{n \rightarrow \infty} \frac{1}{n} \sum_{j=0}^{j=n-1} f\left(T^{j} x\right)=f^{*}(x)
$$

for $\mu$-a.e. $x \in X$ and in $L^{1}$.

Where $f^{*} \in L_{\mu}^{1}(x)$ is $T$-invariant $(f(T x)=f(x))$ and $\int_{X} f^{*} d \mu=\int_{X} f d \mu$.

Similary for a measure preserving flow $\phi_{t}$ :

$$
\lim _{|b-a| \rightarrow \infty} \frac{1}{b-a} \int_{a}^{b} f\left(\phi_{t} x\right) d t=f^{*}(x)
$$

A weaker version of this theorem (Mean Ergodic Theorem) for $L^{2}$ functions with convergence in $L^{2}$ was proved earlier by Von Neumann.

In general although orbit averages exists $\mu$-a.e. they are not necessarily equal to the integral of the function. For example consider the identity transformation on a non-trivial probability space and any $L^{1}$ function which is not a.e. constant.

We wish to study the situation when the orbit averages do coincide with the measure integral.

Definition 2. A $T$-invariant measure is ergodic if for all measurable $A$ :

$$
T^{-1}(A)=A \Longrightarrow \mu(A) \in\{0,1\}
$$

ergodicity is equivalent to many natural properties of a MPT in particular the following:

Proposition 1. A $T$-invariant measure is ergodic if and only if every measurable $T$-invariant function is constant a.e.

A direct corollary is that for an ergodic MPT the orbit average $f^{*}$ of the PET is constant a.e. and equal to the integral.

The notion of ergodicity can be further developed into the strictly stronger properties of weak-mixing and mixing. See [1],[2] for details.

\section{ExAMPLES}

2.1. Hamiltonian Flow. Liouville's theorem tells us that the Liouville measure is invariant under the Hamiltonian flow. In particular, as was shown by Hopf, the geodesic flow on the unit tangent bundle of a compact Riemannian manifold of negative sectional curvature is ergodic.

2.2. Toral Shift. Given an n-dimensional torus $\mathbb{T}^{n}=\mathbb{Z}^{n} \backslash \mathbb{R}^{n}$ a shift is a transformation $T(x)=x+\alpha(\bmod 1)$ for some $\alpha \in \mathbb{R}^{n}$. This system is ergodic w.r.t. the Haar measure iff $1, \alpha_{1}, \alpha_{2}, \ldots, \alpha_{n}$ are rationally independant, e.g. a onedimensional circle rotation $x \mapsto x+\alpha(\bmod 1)$ is ergodic iff $\alpha$ is irrational. 
2.3. Toral Automorphism. Let $A \in \mathrm{GL}_{n}(\mathbb{Z})$ be an automorphism of the torus $\mathbb{T}^{n}$. A preserves the Haar measure $(|\operatorname{det}(A)|=1)$ and is ergodic iff it is quasihyperbolic: no eigenvalues which are roots of unity. Specifically hyperbolic toral automorphisms (no eigenvalues of modulus 1) are ergodic. Hyperbolic toral automorphims are also colloquially called "Cat Maps" following Arnold's example.

\section{Continuous Transformations on Compact Metric Spaces}

An important question for a transformation or a flow on a measure space is whether an invariant probability measure necessarily exists. For continuous actions on compact metric spaces the Krylov-Bogoliubov theorem guarantees us that an invariant Borel measure always exists. Consequentially, we will be interested in the following notion.

Definition 3. Given a continuous MPT $T: X \rightarrow X$ of a compact metric space $\mathrm{X}$ let $\mathcal{M}^{T}(X)$ be the space of all $T$-invariant Borel probability measures on $\mathrm{X}$ endorsed with the weak-* topology (as inherited from the dual of $C(X)$ ).

$\mathcal{M}^{T}(X)$ is non-empty, convex, $T_{*}$-invariant and weak-* compact. The extreme points of $\mathcal{M}^{T}(X)$ are exactly the ergodic measures, so as $\mathcal{M}^{T}(X) \neq \emptyset$ we must have an ergodic T-invariant measure as well. This leads us to the following important result which can be proved using Choquet theory or conditional measures.

Ergodic Decomposition. Given a continuous MPT $T: X \rightarrow X$ of a compact metric space $\mathrm{X}$ let $\mathcal{E}^{T}(X) \subseteq \mathcal{M}^{T}(X)$ be the set of all ergodic T-invariant Borel probability measures. For every $\mu \in \mathcal{M}^{T}(X)$ there is a Borel measure $\nu$ on $\mathcal{E}^{T}(X)$ so that $\forall f \in C(X): \int_{X} f d \mu=\int_{\mathcal{E}^{T}(X)} \int_{X} f d \eta d \nu(\eta)$, i.e. every T-invariant probability measure is a generalized convex combination of ergodic ones.

The ergodic decomposition is especially useful at reducing questions about invariant measure to questions about ergodic invariant measures.

Let us consider the situation when $\mathcal{M}^{T}(X)$ consists of a single measure, such a system is called "Uniquely Ergodic". This is the case, for example, for an ergodic shift on the torus but not for the geodesic flow of negative curvature or for the ergodic toral automorphism. The following is an easy consequence of the definition:

Proposition 2. Let $T: X \rightarrow X$ be a continuous uniquely ergodic MPT of a compact metric space then $\forall x \in X$ :

$$
\lim _{n \rightarrow \infty} \frac{1}{n} \sum_{j=0}^{j=n-1} f\left(T^{j} x\right)=\int_{X} f d \mu,
$$

where $\mu$ is the unique $T$-invariant measure.

Notice that in this case the convergence is for all orbit averages. Such results which describe the orbit averages of all points are especially useful in applications in number theory. The question of classification of all orbit averages in nonuniquely ergodic systems is related to measure rigidity and is the subject of ongoing research. Measure rigidity is central to the proof of Arithmetic Quantum Unique Ergodicity. 


\section{ENTROPY}

Entropy is an important invariant of a MPT which is of great use in the study of Quantum Ergodicity. Roughly speaking entropy mesures the exponential complexity of the orbits, this statement is made explicit in the Brin-Katok theorem.

Definition 4. Given a countable mesurable paritition $\boldsymbol{\xi}$ of a probability space $(X, \mathcal{B}, \mu)$ we define the entropy according to Shannon as:

$$
H_{\mu}(\boldsymbol{\xi})=-\sum_{j=0}^{j=\infty} \mu\left(\xi_{j}\right) \log \left(\mu\left(\xi_{j}\right)\right)
$$

Given two such partitions $\boldsymbol{\xi}, \boldsymbol{\eta}$ we define the conditional entropy:

$$
H_{\mu}(\boldsymbol{\xi} \mid \boldsymbol{\eta})=\sum_{j=1}^{j=\infty} \mu\left(\eta_{j}\right) H_{\mu}\left(\frac{\mu\left(\xi_{1} \cap \eta_{j}\right)}{\mu\left(\eta_{j}\right)}, \frac{\mu\left(\xi_{2} \cap \eta_{j}\right)}{\mu\left(\eta_{j}\right)}, \ldots\right)
$$

Notice that the entropy of a partition is non-negative yet might be infinite.

We leave the statement of the standard properties of the partition entropy to the references [1], [3]. A useful interpretation of the entropy is in terms of measurement. If the partition $\boldsymbol{\xi}$ describes the resolution of our measurement, then the entropy $H_{\mu}(\boldsymbol{\xi})$ is a related to the amount of information we gain by the measurement.

The trivial paritition $\{\emptyset, X\}$ has zero entropy, while for a partition with $k$ elements we have the convexity inequality $H_{\mu}(\boldsymbol{\xi}) \leq \log (k)$ with equality iff the partition has equal distribution of mass: $\forall 1 \leq j \leq k: \mu\left(\xi_{j}\right)=\frac{1}{k}$. This simple statement is a prototype for many results regarding classification of the measure of maximal entropy.

We now turn to define the Kolmogorov-Sinai entropy of a MPT.

Definition 5. Given a MPT $T: X \rightarrow X$ w.r.t. $\mu$ and a countable measurable partition $\boldsymbol{\xi}$ define $h_{\mu}(T, \boldsymbol{\xi})=\lim _{n \rightarrow \infty} \frac{1}{n} H_{\mu}\left(\bigvee_{j=0}^{n-1} T^{-j} \boldsymbol{\xi}\right)$. The $\vee$ operator denotes the mutual refinement of partitions.

Now we can define the entropy of a measure preserving transformation:

$$
h_{\mu}(T)=\sup _{\boldsymbol{\xi}: H_{\mu}(\boldsymbol{\xi})<\infty} h_{\mu}(T, \boldsymbol{\xi})
$$

where the supremum is taken over all measurable countable partition with finite entropy.

In the measurement interpretation $H_{\mu}\left(\bigvee_{j=0}^{n-1} T^{-j} \boldsymbol{\xi}\right)$ can be explained as the total information gained using $\boldsymbol{\xi}$-measurement in the times $0 \leq j<n$. Note that the supremum definition of $h_{\mu}(T)$ makes it a measurable invariant of MPTs.

The Kolmogorov-Sinai theorem allows us to reduce the calculation of $h_{\mu}(T)$ to the calculation of $h_{\mu}(T, \boldsymbol{\xi})$ for a special partition $\boldsymbol{\xi}$ which is called generating. See the references [1], [3] for details.

To state the important SMB theorem we first need the following definition. 
Definition 6. The information function is $I_{\mu}[\boldsymbol{\xi}](x)=-\log \left(\mu\left([x]_{\xi}\right)\right)$ where $[x]_{\xi}$ is the atom of $x$ - the partition element of $\boldsymbol{\xi}$ which contains $x$. Similarly define $I_{\mu}[\boldsymbol{\xi} \mid \boldsymbol{\eta}](x)=-\log \left(\frac{\mu\left([x]_{\xi \vee \eta}\right)}{\mu\left([x]_{\eta}\right)}\right)$.

It is easy to see that $H_{\mu}(\boldsymbol{\xi})=\int_{X} I_{\mu}[\boldsymbol{\xi}] d \mu$ and $H_{\mu}(\boldsymbol{\xi} \mid \boldsymbol{\eta})=\int_{X} I_{\mu}[\boldsymbol{\xi} \mid \boldsymbol{\eta}] d \mu$.

The SMB theorem explains the asymptotical growth of the measure of an atom in a partition under the action of $T$ in terms of entropy. For simplicity we state it only for the ergodic case.

Shannon-McMillan-Briemann Theorem. Let $T: X \rightarrow X$ and $\mu T$-invariant and ergodic, and let $\boldsymbol{\xi}$ be a countable measurable partition so that $H_{\mu}(\boldsymbol{\xi})<\infty$. Then

$$
\lim _{n \rightarrow \infty} \frac{1}{n} I_{\mu}\left[\bigvee_{j=0}^{n-1} T^{-j} \boldsymbol{\xi}\right](x)=h_{\mu}(T, \boldsymbol{\xi})
$$

for $\mu$-a.e $x$ and in $L^{1}$.

This theorem about partition entropy has a parallel regarding transformation entropy which uses the notion of a Bowen ball.

Brin-Katok Theorem. Let $T: X \rightarrow X$ be a homeomorphism of a compact metric space $(X, d)$ and $\mu \in \mathcal{E}^{T}(X)$. For $x \in X, r>0$ and $n \in \mathbb{N}$ define the $(r, n)$ Bowen ball centered at $x$ :

$$
B_{T}(x, r, n)=\left\{y \in X \mid d\left(T^{j} x, T^{j} y\right)<r, \forall 0 \leq j<n\right\}
$$

Then

$$
\lim _{r \rightarrow 0} \limsup _{n \rightarrow \infty} \frac{-\log \left(\mu\left(B_{T}(x, r, n)\right)\right)}{n}=h_{\mu}(T)
$$

For $\mu$-a.e $x$. The same statement is true for the liminf.

\section{REFERENCES}

[1] A. Katok, B. Hasselblat, Introduction to the Modern Theory of Dynamical Systems, Cambridge University Press (1995)

[2] M. Einsiedler, T. Ward, Ergodic Theory with a view towards Number Theory, Springer (2011)

[3] M. Einsiedler, E. Lindenstrauss, T. Ward Entropy in ergodic theory and homogeneous dynamics, Preprint

\section{Hyperbolic Dynamics \\ DIERK SCHLEICHER}

The purpose of this presentation is to give an overview on hyperbolic dynamical systems, covering some of the basic definitions and then some properties for geodesic flows on manifolds with negative curvature. 


\section{Hyperbolic Sets}

Definition 1. Consider the fixed point 0 of the linear automorphism $x \mapsto A x$ for $x \in \mathbb{R}^{n}$ and an $n \times n$-matrix $A$. This fixed point is hyperbolic if there is a splitting $\mathbb{R}^{n}=E^{s} \oplus E^{u}$ and a $\lambda>1$ so that, for an appropriate norm on $\mathbb{R}^{n}$, we have

$$
\left\|A v^{s}\right\|<\lambda\left\|v^{s}\right\| \quad \text { and } \quad\left\|A^{-1} v^{u}\right\|<\lambda\left\|v^{u}\right\|
$$

for all non-zero $v^{s} \in E^{s}$ and $v^{u} \in E^{u}$, and so that the subspaces are $A$-invariant:

$$
A E^{s}=E^{s} \text { and } A E^{u}=E^{u} .
$$

$E^{s}$ is called the stable subspace and $E^{u}$ is called the unstable subspace.

Remark 1. a) The degenerate case that $E^{s}$ or $E^{u}$ are the null spaces are admitted, so that $A$ is uniformly stable or uniformly unstable.

b) The simplest case is if $A$ is diagonal and all eigenvalues have absolute values different from 1.

c) It might seem more natural to write the condition in the unstable subspace as $\left\|A v^{u}\right\|>(1 / \lambda)\left\|v^{u}\right\|$; however, this does not work for at least two reasons: for one, expansion in the forward direction happens in the complement of $E^{s}$, which is much bigger than $E^{u}$; and for another, we usually do not have control in the forward direction of unstable vectors (in less general settings than that of a vector space), for instance we might be on a compact space where there are limits for expansion.

d) If we use a "wrong" norm on $\mathbb{R}^{n}$, then the hyperbolicity condition might be violated. However, the following dynamical condition will still hold for any norm: there are $\lambda<1$ and $C>0$ so that

$$
\left\|A^{n} v^{s}\right\|<C \lambda^{n}\left\|v^{s}\right\| \quad \text { and } \quad\left\|A^{-n} v^{u}\right\|<C \lambda^{n}\left\|v^{u}\right\|
$$

for all $n \geq 0$.

Example 1 (Hyperbolic Toral Automorphism). If $A \in \mathrm{SL}_{N}(\mathbb{Z})$, then $x \mapsto A x$ descends to an automorphism of the quotient torus $A:\left(\mathbb{R}^{N} / \mathbb{Z}^{N}\right) \rightarrow\left(\mathbb{R}^{N} / \mathbb{Z}^{N}\right)$ with a fixed point at 0 . Locally, the dynamics of this fixed point is the same as that of the linear map $A: \mathbb{R}^{N} \rightarrow \mathbb{R}^{N}$.

Hyperbolic toral automorphisms are also known as "Arnol'd's Cat Maps" because their dynamics is sometimes illustrated on a picture of a cat.

Definition 2. Suppose $f: M \rightarrow M$ is a smooth diffeomorphism of a smooth manifold and $p$ is a fixed point of $f$. Then $p$ is a hyperbolic fixed point of $f$ if there is an invariant splitting of $T_{p} M=E_{p}^{s} \oplus E_{p}^{u}$ and a $\lambda>0$ so that for some norm on $T_{p}$ we have again

$$
\left\|d f v^{s}\right\|<\lambda\left\|v^{s}\right\| \quad \text { and } \quad\left\|d f^{-1} v^{u}\right\|<\lambda\left\|v^{u}\right\|
$$

for all non-zero $v^{s} \in E_{p}^{s}$ and $v^{u} \in E_{p}^{u}$, and so that the subspaces are $d f$-invariant:

$$
d f E_{p}^{s}=E_{p}^{s} \quad \text { and } \quad d f E_{p}^{u}=E_{p}^{u} .
$$


The same definition also applies to periodic points (where $f$ is replaced by the first return map).

Definition 3 (Hyperbolic Set on Manifold). Consider a smooth diffeomorphism $f: M \rightarrow M$ on a smooth Riemannian manifold $M$. A compact invariant set $\Lambda \subset M$ is called a hyperbolic set of $M$ if there exists a continuous splitting of all tangent spaces $T_{p} M=E_{p}^{s} \oplus E_{p}^{u}$, for $p \in \Lambda$, and $\lambda<1, C>0$ so that again

$$
\left\|d f^{n} v^{s}\right\|<C \lambda^{n}\left\|v^{s}\right\| \quad \text { and } \quad\left\|d f^{-n} v^{u}\right\|<C \lambda^{n}\left\|v^{u}\right\|
$$

for all non-zero $v^{s} \in E_{p}^{s}$ and $v^{u} \in E_{p}^{u}$ and $n \geq 0$, and so that the subspaces are $d f$-invariant:

$$
d f E_{p}^{s}=E_{f(p)}^{s} \quad \text { and } \quad d f^{-1} E_{p}^{u}=E_{f^{-1}(p)}^{u} .
$$

The families of spaces $E_{p}^{s}$ and $E_{p}^{u}$ are called the stable and unstable distributions.

Remark 2 (Adapted Metric). A Riemannian metric on $M$ is called an adapted (or Lyapunov) metric if it satisfies the following stronger condition

$$
\left\|d f v^{s}\right\|<\lambda\left\|v^{s}\right\| \text { and } \quad\left\|d f^{-1} v^{u}\right\|<\lambda\left\|v^{u}\right\| .
$$

One can always modify the metric in a neighborhood of $\Lambda$ so that this condition is satisfied (by slightly increasing $\lambda$ ).

A smooth diffeomorphism is called an Anosov diffeomorphism if $M$ is compact and $\Lambda=M$ is a hyperbolic set for $f$.

Remark 3 (Cone Condition). In practice, hyperbolicity is often established most easily using a cone condition: there are continuous families $\tilde{E}_{p}^{s}$ and $\tilde{E}_{p}^{u}$ of tangent subspaces with $T_{p} M=\tilde{E}_{p}^{s} \oplus \tilde{E}_{p}^{u}$ and $\alpha<1$ and cones

$$
\begin{gathered}
C_{p, \alpha}^{s}:=\left\{x \in T_{p}: x=x^{s}+x^{u}, x^{s} \in \tilde{E}_{p}^{s}, x^{u} \in \tilde{E}_{p}^{u},\left\|x^{u}\right\|<\alpha\left\|x^{s}\right\|\right\}, \\
C_{p, \alpha}^{u}:=\left\{x \in T_{p}: x=x^{s}+x^{u}, x^{s} \in \tilde{E}_{p}^{s}, x^{u} \in \tilde{E}_{p}^{u},\left\|x^{s}\right\|<\alpha\left\|x^{u}\right\|\right\}
\end{gathered}
$$

so that $d f\left(C_{p}^{u}\right) \subset C_{f(p)}^{u}$ and $d f^{-1}\left(C_{p}^{s}\right) \subset C_{f^{-1}(p)}^{s}$ for all $p$ (invariance) and $\left\|d f_{p} x\right\|<$ $\|x\|$ for non-zero $x \in C_{p, \alpha}^{s},\left\|d f_{p}^{-1} x\right\|<\|x\|$ for non-zero $x \in C_{p, \alpha}^{u}$ (contraction).

\section{Properties of Hyperbolic Dynamics}

Definition 4 (Pseudo-orbits, Shadowing). If $f: M \rightarrow M$ is a map on a metric space $(M, d)$, then a $\delta$-pseudoorbit is a sequence $\left(x_{n}\right) \subset M$ with $d\left(x_{n+1}, f\left(x_{n}\right)\right)<\delta$ for all $n$. The orbit of $x$ is said to $\varepsilon$-shadow the pseudoorbit $\left(x_{n}\right)$ if $d\left(f^{n}(x), x_{n}\right)<\varepsilon$ for all $n$.

In practice, any numerically calculated orbit of a dynamical system is really a $\delta$-pseudoorbit, where $\delta$ depends on the accuracy of the calculation. There is no hope that a $\delta$-pseudoorbit $\left(x_{n}\right)$ remains close to the actual orbit of $x_{0}$ (unless the unstable direction is 0-dimensional), but there $i s$ hope that it remains close to an actual orbit of a nearby point. This is the idea of shadowing, and the theorem is as follows. 
Theorem 1 (The Shadowing Theorem). Let $f: M \rightarrow M$ be a smooth hyperbolic (Anosov) dynamical system on a smooth Riemannian manifold. Then for every $\varepsilon>0$ there is a $\delta>0$ so that any $\delta$-pseudoorbit is $\varepsilon$-shadowed by an actual orbit.

The next definition is structural stability. While shadowing concerns insensitivity with respect to initial conditions, structural stability concerns insensitivity with respect to the (the parameters of) the actual map.

Definition 5 (Structural Stability). A smooth Anosov diffeomorphism $f: M \rightarrow$ $M$ on a compact smooth Riemannian manifold is called structurally stable if there is an $\varepsilon>0$ so that any smooth diffeomorphism $g: M \rightarrow M$ that is uniformly $\varepsilon$-close to $f$ is topologically conjugate to $f$ (i.e., there is a homeomorphism $\phi: M \rightarrow M$ with $\phi \circ f=g \circ \phi)$.

Theorem 2 (Hyperbolic Maps are Structurally Stable). Hyperbolic maps are structurally stable.

A key idea in the proof is shadowing: if $g$ is uniformly $\varepsilon$-close to $f$, then any actual orbit of $g$ is an $\varepsilon$-pseudoorbit of $f$ (and vice versa), and this defines a homeomorphism $\phi: M \rightarrow M$ so that the actual orbit of $x$ under $g$ is shadowed by the actual orbit of $\phi(x)$.

Theorem 3 (The Stable Manifold Theorem). Let $f: M \rightarrow M$ be a hyperbolic (Anosov) dynamical system on a smooth Riemannian manifold, where we assume that the metric is adapted. Then every $p \in M$ has $C^{1}$-manifolds $W_{p}^{s}, W_{p}^{u}$, called the local stable and unstable manifolds, with the following properties:

(1) for $p \in M$, the local stable manifold, defined as

$$
W_{p, \varepsilon}^{s}:=\left\{x \in M: d\left(f^{n}(x), f^{n}(p)\right)<\varepsilon \text { for all } n \geq 0\right\},
$$

is an embedded $C^{1}$-disk;

(2) all $q \in W_{p, \varepsilon}^{s}$ have $T_{q} W_{p}^{s}=E_{q}^{s}$;

(3) $f\left(W_{p, \varepsilon}^{s}\right) \subset W_{f(p), \varepsilon}^{s} ; f^{-1}\left(W_{p}^{u}\right) \subset W_{f^{-1}(p)}^{u}$

(4) if $q \in W_{p, \varepsilon}^{s}$, then $W_{q, \alpha}^{s} \subset W_{p, \varepsilon}^{s}$ for some $\alpha>0$;

(5) $f: W_{p, \varepsilon}^{s} \rightarrow W_{f(p), \varepsilon}^{s}$ contracts distances (measured within the local stable manifolds);

and the analogous conditions hold for the local unstable manifolds $W_{p, \varepsilon}^{u}$.

In fact, one can extend these local stable and unstable manifolds to global manifolds using

$$
W_{p}^{s}:=\bigcup_{n \geq 0} f^{-n}\left(W_{f^{n}(p), \varepsilon}^{s}\right) \quad \text { and } \quad W_{p}^{u} \bigcup_{n \geq 0} f^{n}\left(W_{f^{-n}(p), \varepsilon}^{u}\right)
$$

for appropriate $\varepsilon>0$. We have $W_{p}^{s}=\left\{x \in M: d\left(f^{n}(x), f^{n}(p)\right) \rightarrow 0\right\}$ and $W_{p}^{u}=\left\{x \in M: d\left(f^{-n}(x), f^{-n}(p)\right) \rightarrow 0\right\}$. 


\section{Geodesic Flow on Negatively Curved Manifolds}

A geodesic flow on a Riemannian manifold $M$ is called hyperbolic if there is a splitting of the unit tangent space $S_{p} M=E^{0} \oplus E^{s} \oplus E^{u}$, where $E^{0}$ is the onedimensional flow direction (along which there is no expansion or contraction) and the spaces $E^{s}$ and $E^{u}$ are similar as before. Phase space is the unit tangent bundle $S M$ of dimension $2 n-1$ if $n$ is the dimension of $M$.

Theorem 4. The geodesic flow on compact manifolds with bounded negative curvature is hyperbolic.

The stable and unstable directions both have dimension $n-1$.

Periodic dynamics is often of particular importance: periodic orbits are easiest to understand, and in many cases they determine the global properties of a hyperbolic dynamical system. We give two examples for this observation: periodic orbits are dense (so that every orbit is approximated arbitrarily well by a periodic orbit), and there exists a (non-periodic) orbit that is dense in phase space.

Theorem 5. For the geodesic flow on a hyperbolic manifold, periodic orbits are dense.

Theorem 6 (Existence of Dense Orbit). For the geodesic flow on hyperbolic manifolds there is a dense orbit.

Proposition 1 (Topological Transitivity). If a flow (or a map) on a second countable space has the property that for any two open sets $U$ and $V$, the dynamics maps $U$ to an open set with non-empty intersection with $V$, then there is a dense orbit.

Remark 4 (Topological Transitivity). Topological transitivity of a dynamical system seems to indicate transitive action on open sets, but is often defined as the existence of a dense orbit. The lemma says that there often is not much of a difference (the converse is obvious).

Proof. For notational convenience, we give the proof for a map $f$; the argument for a flow is the same. Let $\left(U_{n}\right)_{n \in \mathbb{N}}$ be a countable basis of the topology. We will construct an orbit that visits all $U_{n}$. First set $V_{0}:=U_{0}$. Then there is an $n_{1}$ so that $f^{n_{1}}\left(V_{0}\right)$ intersects $U_{1}$; let $V_{1}:=f^{-n_{1}}\left(U_{1} \cap f^{n_{1}}\left(V_{0}\right)\right) \subset V_{0}$. Similarly, for $k>1$ there is an $n_{k}$ so that $f^{n_{k}}\left(V_{k-1}\right)$ intersects $U_{k}$; let $V_{k}:=f^{-n_{k}}\left(U_{k} \cap f^{n_{k}}\left(V_{k}\right)\right) \subset V_{k-1}$. Now let $K:=\bigcap_{k \geq 0} \overline{V_{k}}$. Any orbit starting in $K$ will visit the closures of all open sets, hence all open sets (on reasonable spaces).

\section{REFERENCES}

$[\mathrm{KH}]$ Anatol Katok and Boris Hasselblatt, Introduction to the modern theory of dynamical systems, Cambridge University Press, 1995.

[BS] Michael Brin and G. Stuck, Introduction to Dynamical Systems, Cambridge University Press, 2002. 


\section{Quantum ergodicity for smooth Hamiltonian flow INGO WiTT}

This talk closely followed lecture notes by EvAns and Zworski [2, Chap. 14]. Let $(M, g)$ be a compact $C^{\infty}$ Riemannian manifold; $d=\operatorname{dim} M$. We consider the Schrödinger operator $P(h)=-h^{2} \Delta+V(x)$ as $h \rightarrow+0$, where $\Delta$ is the LaplaceBeltrami operator on $M$ and $V \in C^{\infty}(M ; \mathbb{R})$. It is known that the spectrum of $P(h)$ is discrete. Let $\left\{u_{j}(h)\right\}_{j \in \mathbb{N}}$ be an orthonormal basis of $L^{2}\left(M, d V_{g}\right)$ consisting of eigenfunctions of $P(h)$ and $\left\{E_{j}(h)\right\}_{j \in \mathbb{N}}$ be the associated sequence of eigenvalues, where $E_{1}(h) \leq E_{2}(h) \leq \ldots$ and $E_{j}(h) \rightarrow \infty$ as $j \rightarrow \infty$. Regarding $\{P(h)\}_{h>0}$ as a semiclassical operator, its principal symbol is $p(x, \xi)=|\xi|^{2}+V(x)$. The latter is the classical energy of the system under consideration.

We make the following assumptions: Fix real numbers $a<b$.

(A) The interval $[a, b]$ consists of regular values for $p(x, \xi)$. This means that $(\nabla V)(x) \neq 0$ whenever $x \in V^{-1}([a, b])$.

(B) For each $c \in[a, b]$, the Hamilton flow $\left\{\exp \left(t H_{p}\right)\right\}_{t \in \mathbb{R}}$ restricted to the energy shell $\Sigma_{c}=p^{-1}(c)$ is ergodic with respect to the Liouville measure $\mu_{c}$.

Remark. Assumption (A) means that $p^{-1}([a, b])=\bigcup_{a \leq c \leq b} \Sigma_{c}$ is foliated by the compact hypersurfaces $\Sigma_{c}$. On each $\Sigma_{c}$, one then has the Liouville measure $\mu_{c}$ given by the property that, for all continuous functions $f: p^{-1}([a, b]) \rightarrow \mathbb{R}$,

$$
\int_{p^{-1}([a, b])} f d x d \xi=\int_{a}^{b}\left(\left.\int_{\Sigma_{c}} f\right|_{\Sigma_{c}} d \mu_{c}\right) d c
$$

with $d x d \xi$ being the symplectic volume. It is not hard to see that the measures $\mu_{c}$ are invariant for $\left\{\exp \left(t H_{p}\right)\right\}_{t \in \mathbb{R}}$ which then leads to assumption (B).

The first main result of this talk was:

Theorem 1. Under the assumptions (A) and (B) above, one has, for each semiclassical pseudodifferential operator $A$ of order zero such that $\alpha(A, c)=\frac{\int_{\Sigma_{c}} \sigma^{0}(A) d \mu_{c}}{\int_{\Sigma_{c}} d \mu_{c}}$ is independent of $c \in[a, b]$,

$$
(2 \pi h)^{d} \sum_{j: a \leq E_{j} \leq b}\left|\left\langle A u_{j}, u_{j}\right\rangle-\alpha(A)\right|^{2} \rightarrow 0 \quad \text { as } h \rightarrow+0
$$

In order to prove Theorem 1, one needs two results which were discussed in previous talks:

Proposition 1 (Generalized Weyl's law). For each semiclassical pseudodifferential operator $A$ of order zero, one has

$$
\begin{aligned}
(2 \pi h)^{d} \sum_{j: a \leq E_{j} \leq b}\left\langle A u_{j}, u_{j}\right\rangle \rightarrow \int_{p^{-1}([a, b])} & \sigma^{0}(A) d x d \xi \\
& =\int_{a}^{b} \mu_{c}\left(\Sigma_{c}\right) \alpha(A, c) d c \quad \text { as } h \rightarrow+0 .
\end{aligned}
$$


Proof. See the talk by C. Neira Jiménez. Note that here and in the sequel we tactically assume that the limit of $\sigma^{0}(A)$ as $h \rightarrow+0$ exists, and then this limit is used in the right-hand side of (2).

Remark. (i) For $A$ the identity, one obtains Weyl's law

$$
(2 \pi h)^{d} \sharp\left\{j \mid a \leq E_{j} \leq b\right\} \rightarrow \int_{p^{-1}([a, b])} d x d \xi=\int_{a}^{b} \mu_{c}\left(\Sigma_{c}\right) d c \quad \text { as } h \rightarrow+0,
$$

where the right-hand side is the volume of $p^{-1}([a, b])$.

(ii) Let $\Pi_{[a, b]}(h)$ be the spectral projection for $P(h)$ corresponding to the spectral interval $[a, b]$. Then (2) becomes the statement that, for $A$ as in Theorem 1 ,

$$
(2 \pi h)^{d} \operatorname{tr}\left(A \Pi_{[a, b]}(h)\right) \rightarrow \int_{p^{-1}([a, b])} \sigma^{0}(A) d x d \xi=\alpha(A) \int_{a}^{b} \mu_{c}\left(\Sigma_{c}\right) d c \quad \text { as } h \rightarrow+0
$$

while assumptions (A) and (B) together yield the stronger result

$$
(2 \pi h)^{d}\left\|(A-\alpha(A)) \Pi_{[a, b]}(h)\right\|_{\mathrm{HS}}^{2} \rightarrow 0 \quad \text { as } h \rightarrow+0,
$$

with \|\|$_{\text {HS }}$ being the Hilbert-Schmidt norm, for the class of observables $A$ as in the theorem. Thus seeing (2) as a statement about the expectation value for the quantum system to observe $A$ in the energy range $[a, b]$, the expression occurring in the left-hand side of (1) is interpreted as quantum variance.

The next result will allow us to utilize the ergodicity assumption. To state this result, recall that, in the semiclassical setting, one has a short exact sequence

$$
0 \rightarrow h \Psi^{m-1}(M) \rightarrow \Psi^{m}(M) \stackrel{\sigma^{m}}{\longrightarrow} S^{m}\left(T^{*} M\right) / h S^{m-1}\left(T^{*} M\right) \rightarrow 0
$$

which splits. Fix a splitting $\mathrm{Op}^{m}: S^{m}\left(T^{*} M\right) / h S^{m-1}\left(T^{*} M\right) \rightarrow \Psi^{m}(M)$.

Proposition 2 (Weak form of Egorov's theorem). Let $A$ be a semiclassical pseudodifferential operator of order zero. Let $A(t)=e^{i t P(h) / h} A e^{-i t P(h) / h}, t \in \mathbb{R}$, be the evolution of the observable $A$ in the Heisenberg picture. Then

$$
\left\|A(t)-\mathrm{Op}^{0}\left(\exp \left(t H_{p}\right)^{*} \sigma^{0}(A)\right)\right\|_{L^{2}\left(\mathbb{R}^{d}\right) \rightarrow L^{2}\left(\mathbb{R}^{d}\right)}=O(h)
$$

holds locally uniformly in $t$.

Proof. See the talk by B. Ammann.

We shall demonstrate the proof of Theorem 1, as it sets out the basic technique which then was used in later talks.

Proof of Theorem 1. Take $\alpha(A)$ like in the statement of the theorem, it is independent of $c \in[a, b]$ by assumption. Replacing $A$ with $A-\alpha(A) I$, one can assume that $\alpha(A)=0$.

Define

$$
\epsilon(h)=(2 \pi h)^{d} \sum_{j: a \leq E_{j} \leq b}\left|\left\langle A u_{j}, u_{j}\right\rangle\right|^{2} .
$$

We need to show that $\epsilon(h) \rightarrow 0$ as $h \rightarrow+0$. This is achieved as follows: 
One has

$$
\begin{aligned}
\left\langle A u_{j}, u_{j}\right\rangle=\left\langle A e^{-i t E_{j} / h} u_{j}, e^{-i t E_{j} / h} u_{j}\right\rangle & \\
& =\left\langle A e^{-i t P(h) / h} u_{j}, e^{-i t P(h) / h} u_{j}\right\rangle=\left\langle A(t) u_{j}, u_{j}\right\rangle, \quad \forall t \in \mathbb{R} .
\end{aligned}
$$

With the notation $\langle A\rangle_{T}=T^{-1} \int_{0}^{T} A(t) d t, T>0$, averaging over $t$ yields

$$
\left\langle A u_{j}, u_{j}\right\rangle=\left\langle\langle A\rangle_{T} u_{j}, u_{j}\right\rangle
$$

i.e.,

$$
\left|\left\langle A u_{j}, u_{j}\right\rangle\right|^{2}=\left|\left\langle\langle A\rangle_{T} u_{j}, u_{j}\right\rangle\right|^{2} \leq\left\|\langle A\rangle_{T} u_{j}\right\|^{2}=\left\langle\left\langle A^{*}\right\rangle_{T}\langle A\rangle_{T} u_{j}, u_{j}\right\rangle
$$

as $\left\|u_{j}\right\|=1$. Hence,

$$
\epsilon(h) \leq(2 \pi h)^{d} \sum_{j: a \leq E_{j} \leq b}\left\langle\left\langle A^{*}\right\rangle_{T}\langle A\rangle_{T} u_{j}, u_{j}\right\rangle .
$$

By Proposition 2, denoting $\tilde{A}(t)=\mathrm{Op}^{0}\left(\exp \left(t H_{p}\right)^{*} \sigma^{0}(A)\right)$, one has

$$
\left\|\langle A\rangle_{T}-\langle\tilde{A}\rangle_{T}\right\|_{L^{2}\left(\mathbb{R}^{d}\right) \rightarrow L^{2}\left(\mathbb{R}^{d}\right)}=O_{T}(h) \quad \text { as } h \rightarrow+0,
$$

with an error term depending on $T>0$. Note that $\sigma^{0}(\tilde{A}(t))=\exp \left(t H_{p}\right)^{*} \sigma^{0}(A)$ and

$$
\sigma^{0}\left(\langle\tilde{A}\rangle_{T}\right)=T^{-1} \int_{0}^{T} \exp \left(t H_{p}\right)^{*} \sigma^{0}(A) d t
$$

where in the following the right-hand side will be abbreviated as $\left\langle\sigma^{0}(A)\right\rangle_{T}$. By Proposition 1, one then arrives at

$$
\begin{aligned}
\limsup _{h \rightarrow+0} \epsilon(h) & \leq \limsup _{h \rightarrow+0}\left((2 \pi h)^{d} \sum_{j: a \leq E_{j} \leq b}\left\langle\left\langle\tilde{A}^{*}\right\rangle_{T}\langle\tilde{A}\rangle_{T} u_{j}, u_{j}\right\rangle+O_{T}(h)\right) \\
& =\int_{p^{-1}([a, b])} \sigma^{0}\left(\left\langle\tilde{A}^{*}\right\rangle_{T}\langle\tilde{A}\rangle_{T}\right) d x d \xi \\
& =\int_{p^{-1}([a, b])}\left|\sigma^{0}\left(\langle\tilde{A}\rangle_{T}\right)\right|^{2} d x d \xi
\end{aligned}
$$

The ergodicity assumption (and $\alpha(A)=0$ ) now yields that

$$
\int_{p^{-1}([a, b])}\left|\sigma^{0}\left(\langle\tilde{A}\rangle_{T}\right)\right|^{2} d x d \xi=\int_{a}^{b}\left(\int_{\Sigma_{c}}\left|\sigma^{0}\left(\langle\tilde{A}\rangle_{T}\right)\right|^{2} d \mu_{c}\right) d c \rightarrow 0 \quad \text { as } T \rightarrow \infty .
$$

From that one infers that $\limsup _{h \rightarrow+0} \epsilon(h)=0$ which finishes the proof.

A consequence of Theorem 1 is the following result which is commonly refereed to as quantum ergodicity:

Theorem 2. Let assumptions (A) and (B) be fulfilled. Then, for each $h>0$ small, there exists a subset $\Lambda(h) \subseteq\left\{j \mid a \leq E_{j}(h) \leq b\right\}$ with the property that

$$
\lim _{h \rightarrow+0} \frac{\sharp \Lambda(h)}{\sharp\left\{j \mid a \leq E_{j}(h) \leq b\right\}}=1
$$


and such that, for all semiclassical pseudodifferential operator $A$ of order zero satisfying the condition $\alpha(A, c) \equiv \alpha(A)$ stated in Theorem 1, it holds that

$$
\left\langle A u_{j}, u_{j}\right\rangle \rightarrow \alpha(A) \quad \text { as } h \rightarrow+0
$$

for $j \in \Lambda(h)$.

Proof. Consult [2, Chap. 14].

Remark. (i) There is an $h=1$ version of Theorem 2: Let $V=0$. Assume the geodesic flow on $S^{*} M$ be ergodic with respect to the Liouville measure. Then there is a subsequence $\left\{j_{k}\right\}_{k \in \mathbb{N}} \subset \mathbb{N}$ of the sequence of positive integers, of density one, such that

$$
\left\langle A u_{j_{k}}, u_{j_{k}}\right\rangle \rightarrow \frac{1}{\mu_{1}\left(S^{*} M\right)} \int_{S^{*} M} \sigma^{0}(A) d \mu_{1} \quad \text { as } k \rightarrow \infty
$$

holds for all classical pseudodifferential operators $A \in \Psi_{\mathrm{cl}}^{0}(M)$.

(ii) There holds a stronger result than stated in Theorem 2: Let $c \in \mathbb{R}$ be a regular value for $p, \Sigma_{c}=p^{-1}(c)$ be compact, and the Hamilton flow $\left\{\exp \left(t H_{p}\right)\right\}_{t \in \mathbb{R}}$ be ergodic on $\Sigma_{c}$ with respect to the Liouville measure. Then (3) holds as stated for any semiclassical pseudodifferential operator $A$ of order 0 if the interval for the eigenvalues $E_{j}(h)$ is suitably shrunk to $\{c\}$ as $h \rightarrow+0$. For details see [3].

We conclude with some historical comments: The result (4) on quantum ergodicity was announced by ŠNIREL'MAN [4]. The first proof was given by ZELDITCH [6] in case of compact manifolds $M$ of constant negative curvature. The proof of (4) for general compact $M$ with ergodic geodesic flow was given by COLIN DE VERDIÈRE [1]. The idea of the proof as presented here goes back to SunADA [5]. The proof of (ii) of the last remark is due to HELfFER, MARTinez, and Robert [3].

\section{REFERENCES}

[1] Y. Colin de Verdière, Ergodicité et fonctions propres du laplacien. Comm. Math. Phys. 102 (1985), 497-502.

[2] L.C. Evans; M. Zworski, Semiclassical analysis. Lecture notes, Aug. 2011, available from http://math. berkeley.edu/ ${ }^{2}$ worski/semiclassical.pdf.

[3] B. Helffer, A. Martinez, and D. Robert, Ergodicité et limite semi-classique. Comm. Math. Phys. 109 (1987), 313-326.

[4] A.I. Šnirel'man, Ergodic properties of eigenfunctions. Uspehi Mat. Nauk 29 (1974), 181-182 (in Russian).

[5] T. Sunada, Quantum ergodicity. In: Progress in inverse spectral geometry, Trends Math., Birkhäuser, Basel, 1997, pp. 175-196.

[6] S. Zelditch, Uniform distribution of eigenfunctions on compact hyperbolic surfaces. Duke Math. J. 55 (1987), 919-941. 


\section{Quantum Ergodicity for Ergodic Billiards NiLS SCHIPPKUS}

In this talk we considered an extension of a quantum ergodicity result for ergodic geodesic flows on compact manifolds to the more complicated setting of billiard flows on compact manifolds with piecewise smooth boundaries. Examples of these billiards include the Sinai and Stadium Billiard, also (nonconvex) generic polygonal billiards. The main theorem reads as follows:

Theorem 1. Let $(M, g)$ be a compact smooth Riemannian manifold with piecewise smooth boundary. Let $\Delta_{g}$ denote the Dirichlet Laplacian induced by the metric, and $\left\{\psi_{j}\right\}_{j \in \mathbb{N}}$ be an orthonormal basis of eigenfunctions of the Laplacian in $L^{2}\left(S^{*} M\right)$. If the billiard flow $\Phi^{t}$ is ergodic, then there exists a subsequence $\left\{\psi_{j_{k}}\right\}_{k \in \mathbb{N}}$ of density one, such that for any $A \in \Psi_{p h g}^{0}(M)$ we have:

$$
\lim _{k \rightarrow \infty}\left\langle\psi_{j_{k}}, A \psi_{j_{k}}\right\rangle=\int_{S^{*} M} \sigma(A) d \mu
$$

where $\sigma(A)$ denotes the principal symbol of $A$ and where $\mu\left(S^{*} M\right)=1$.

The talk covered in detail the definition of the billiard tables, defined the dynamics in terms of both billiard flow and billiard ball map and treated the problem that $\Phi^{t}$ is not defined on whole $S^{*} M$ but merely a subset of full Liouville measure. In addition, an extension of Egorov's theorem for billiard flows was discussed which is a crucial step in the proof of Theorem 1. As a preparational step it was shown that it suffices to find an extension of this theorem on an even smaller subset that still has full measure in $S^{*} M$ and excludes those orbits that eventually become tangential to the boundary. These orbits are referred to as 'glancing'.

Thus, the complex question of propagation of singularities in directions tangential to the boundary could be avoided and an extension of Egorov's theorem merely needed to deal with transversal reflections, which after a local change of coordinates can be regarded as a standard problem of geometrical optics on a half space $\mathbb{H}^{2 n-1}$ where $n=\operatorname{dim} M$. After these preliminaries, the proof of Theorem 1 follows the argument of the smooth manifold case, with the addition of some basic analysis tools such as cutoffs by partitions of unity in order to avoid the singular and glancing sets.

\section{REFERENCES}

[1] S. Zelditch, M. Zworski "Ergodicity of Eigenfunctions for Ergodic Billiards", Commun. Math. Phys 175, p. 673-682, (1996)

[2] M. Farris "Egorov's theorem on a manifold with diffractive boundary", Commun. P.D.E 6, p.651-687 (1981) 


\section{Quantum Ergodicity for Quantum Maps on the Torus KARSTEN FRITZSCH}

Quantisations of time-discrete dynamical systems with compact phase-space give useful toy models for quantum chaology. Within these, the dynamics generated by maps $\kappa: \mathbb{T}^{2} \longrightarrow \mathbb{T}^{2}, \mathbb{T}^{2}=\mathbb{R}^{2} / \mathbb{Z}^{2}$, constitute an important class.

For position respectively momentum coordinates $x=(q, p)$ consider for instance

i) the hyperbolic toral automorphisms or cat maps:

$$
\kappa(x)=A x \text { for } A \in \mathrm{SL}(2, \mathbb{Z}),|\operatorname{Tr} A|>2
$$

ii) the baker's map:

$$
(q, p) \longmapsto \begin{cases}\left(2 q, \frac{p}{2}\right) & , q \in\left[0, \frac{1}{2}\right) \\ \left(2 q-1, \frac{p+1}{2}\right) & , q \in\left[\frac{1}{2}, 1\right)\end{cases}
$$

Kinematics. (Compare [2, 3, 4] and [7]) Imposing quasi-periodicity conditions in position / momentum representation leads to the quantisation of phase-space,

$$
\mathcal{H}_{\hbar}(\vartheta)=\left\{\psi \in \mathcal{S}^{\prime}(\mathbb{R}) \mid T_{q} \psi=e^{-2 \pi i \vartheta_{2}} \psi, T_{p} \psi=e^{2 \pi i \vartheta_{1}} \psi\right\},
$$

where $T_{q}, T_{p}$ denote unit translations in position respectively momentum. This space turns out to be non-trivial for integer values of $N=\frac{1}{2 \pi \hbar}$ only and the corresponding space $\mathcal{H}_{N}(\vartheta)$ is $N$-dimensional. Following [2] this space will be equipped with the unique inner product such that the translations (in position and momentum) by $\frac{j}{N}$ units act unitarily. This gives a Hilbert space structure with an orthonormal basis given by

$$
e_{j, N}^{\vartheta}=\frac{1}{\sqrt{N}} \sum_{n \in \mathbb{Z}} e^{2 \pi i n \vartheta_{2}} \delta\left(q-n-\frac{j+\vartheta_{1}}{N}\right) \quad, \quad 1 \leq j \leq N .
$$

The translations give rise to a simple expression for the Weyl quantisation of $f \in C^{\infty}\left(\mathbb{T}^{2}\right)$ as given, for instance, in [4]:

$$
\mathrm{Op}_{N}^{\mathrm{w}}(f)=\sum_{k \in \mathbb{Z}^{2}} \hat{f}(k) T\left(\frac{\left(k_{1}, k_{2}\right)}{N}\right)
$$

where $\hat{f}$ denotes the Fourier transform of $f$. The Weyl quantisation of $f$ restricts to an operator $\mathrm{Op}_{N, \vartheta}^{\mathrm{w}}(f): \mathcal{H}_{N}(\vartheta) \longrightarrow \mathcal{H}_{N}(\vartheta)$. (See [2] for detailed arguments.)

Dynamics. Quantisations are neither unique, nor is there a standard quantisation scheme for maps $\mathbb{T}^{2} \longrightarrow \mathbb{T}^{2}$, but there is general agreement upon properties a quantisation should satisfy:

Definition 1. Given a map $\kappa: \mathbb{T}^{2} \longrightarrow \mathbb{T}^{2}$ and $\vartheta \in[0,1)^{2}$, a quantisation of $\kappa$ is a family of unitary operators

$$
U_{N}^{\vartheta}(\kappa): \mathcal{H}_{N}(\vartheta) \longrightarrow \mathcal{H}_{N}(\vartheta)
$$

such that for $N \rightarrow \infty$ an Egorov-type theorem holds:

$$
\left\|U_{N}^{\vartheta}(\kappa)^{-1} \mathrm{Op}_{N, \vartheta}^{\mathrm{w}}(f) U_{N}^{\vartheta}(\kappa)-\mathrm{Op}_{N, \vartheta}^{\mathrm{w}}(f \circ \kappa)\right\|_{\mathcal{L}\left(\mathcal{H}_{N}(\vartheta)\right)} \longrightarrow 0
$$


Alternatively, one could require that a quantisation transforms coherent states in a way related to $\kappa$ or that it is a Fourier integral operator whose phase function is related to a generating function for $\kappa$.

The cat maps are linear, smooth, hyperbolic and ergodic; their quantisations can be constructed by imposing a transformation law with respect to an irreducible representation of the Heisenberg group (see e.g. [2, 3, 5] and [9]): Let $\hat{q} \psi=x \psi$ and $\hat{p} \psi=-i \hbar \partial_{x} \psi$ denote the position respectively momentum operators and

$$
\widetilde{T}\left(k_{1}, k_{2}\right)=e^{i \pi k_{1} k_{2}} e^{-2 \pi i k_{1} \hat{p}} e^{2 \pi i k_{2} \hat{q}} \quad, \quad k_{1}, k_{2} \in \mathbb{Z} .
$$

Then assume

$$
U_{N}^{\vartheta}\left(\kappa_{A}\right)^{*} \widetilde{T}\left(k_{1}, k_{2}\right) U_{N}^{\vartheta}\left(\kappa_{A}\right)=\widetilde{T}\left(\left(k_{1}, k_{2}\right) A^{\dagger}\right)
$$

as well as $U_{N}^{\vartheta}\left(\kappa_{A}\right) \mathcal{H}_{N}(\vartheta) \subset \mathcal{H}_{N}(\vartheta)$. This implies:

i) $A \vartheta-\frac{N}{2}\left(\begin{array}{l}a b \\ c d\end{array}\right)=\vartheta \bmod 1$ for $A=\left(\begin{array}{ll}a & b \\ c & d\end{array}\right)$, which is a condition for quantisation to be possible (cf. $[3,7]$ )

ii) for $k_{1}=k_{2}=0$ unitarity of $U_{N}^{\vartheta}\left(\kappa_{A}\right)$ is obtained

iii) the matrix representing $U_{N}^{\vartheta}\left(\kappa_{A}\right)$ with respect to the basis $e_{j, N}^{\vartheta}$ can be computed explicitly (as done in [3] and [5])

iv) an exact Egorov-type theorem holds (cf. [2] and the detailed exposition of [9]):

$$
U_{N}^{\vartheta}\left(\kappa_{A}\right)^{-1} \mathrm{Op}_{N, \vartheta}^{\mathrm{w}}(f) U_{N}^{\vartheta}\left(\kappa_{A}\right)=\mathrm{Op}_{N, \vartheta}^{\mathrm{w}}\left(f \circ \kappa_{A}\right)
$$

Note that for a given map $\kappa_{A}$ it is not obvious for which values of $\vartheta$ there is a sequence $N_{k} \rightarrow \infty$ of integers such that there is a quantisation for $\kappa_{A}$ on $\mathcal{H}_{N_{k}}(\vartheta)$ for all $k$. For this reason $A$ is assumed to be of a specific form in parts of the literature (cf. [2,3] and [7]).

The baker's map cannot be quantized in this way but one can try to imitate the action of the classical map. This quantisation scheme was introduced by [1] and analysed in detail in [4]. We decompose $\mathcal{H}_{2 N}=\mathcal{H}_{2 N}(0)$ into spaces $\Psi_{N}^{L}, \Psi_{N}^{R}$ which consist of states whose first respectively last $N$ coefficients with respect to the basis $\left\{e_{j, 2 N}\right\}$ vanish. Using the discrete Fourier transform

$$
\left(F_{2 N}\right)_{j k}=\frac{1}{\sqrt{2 N}} e^{-\pi i \frac{k j}{N}}, \quad j, k=1, \ldots, 2 N
$$

the same is done in momentum representation, yielding spaces $\Psi_{N}^{B}$ respectively $\Psi_{N}^{T}$. Then,

$$
B_{2 N}=F_{2 N}^{-1}\left(\begin{array}{cc}
F_{N} & 0 \\
0 & F_{N}
\end{array}\right): \begin{aligned}
& \Psi_{N}^{L} \oplus 0 \longrightarrow \Psi_{N}^{B} \oplus 0 \\
& 0 \oplus \Psi_{N}^{R} \longrightarrow 0 \oplus \Psi_{N}^{T}
\end{aligned}
$$

and the discrete Fourier transform on half of the coordinates, $F_{N}$, incorporates the same compression/dilation properties as does the classical baker's map (cf. [1]). This gives a unitary operator on $\mathcal{H}_{2 N}$ and in [4] it is shown that an Egorov-type theorem holds for "sufficiently nice" functions $f \in C^{\infty}\left(\mathbb{T}^{2}\right)$. 
Quantum Ergodicity. In the more "axiomatic" [8] the following formulation of quantum ergodicity is proven:

Theorem 1. Let $\kappa: \mathbb{T}^{2} \longrightarrow \mathbb{T}^{2}$ be a smooth and ergodic map with quantisation $U_{N}(\kappa)$ and denote the eigenstates of $U_{N}(\kappa)$ by $\varphi_{j, N}$.

a) For all $f \in C^{\infty}\left(\mathbb{T}^{2}\right)$ :

$$
\frac{1}{N} \sum_{j=1}^{N}\left|\left\langle\varphi_{j, N}, \mathrm{Op}_{N}^{\mathrm{w}}(f) \varphi_{j, N}\right\rangle-\int_{\mathbb{T}^{2}} f d \mu\right|^{2} \longrightarrow 0 \quad \text { as } N \rightarrow \infty
$$

b) There is a sequence of sets $J_{N} \subset\{1, \ldots, N\}, N \in \mathbb{N}$ of density 1 such that for all $f \in C^{\infty}\left(\mathbb{T}^{2}\right)$ and all $j: N \mapsto j(N) \in J_{N}$ :

$$
\left\langle\varphi_{j(N), N}, \mathrm{Op}_{N}^{\mathrm{w}}(f) \varphi_{j(N), N}\right\rangle \longrightarrow \int_{\mathbb{T}^{2}} f d \mu \quad \text { as } N \rightarrow \infty
$$

Details on the proof can moreover be found in $[2,4,6,9]$, it goes along the usual lines: Firstly, Egorov and ergodicity are used to prove a), then Chebyshev's inequality is invoked to show the existence of an $f$-dependent sequence $J_{N}(f)$. The dependence on the observable can be removed by a diagonal argument with respect to a basis of $C^{\infty}\left(\mathbb{T}^{2}\right)$.

\section{REFERENCES}

[1] N. L. Balazs and A. Voros, The quantised baker's transformation, Ann. Phys. (NY) 190 (1989) 1-31

[2] A. Bouzouina, and S.De Bivre, Equipartition of the eigenfunctions of quantised ergodic maps on the torus, Commun. Math. Phys. 178 (1996) 83-105

[3] M. Degli Esposti, Quantization of the orientation preserving automorphisms of the torus, Ann. Inst. H. Poincare 58 (1993) 323-341

[4] M. Degli Esposti, S. Nonnenmacher and B. Winn, Quantum variance and ergodicity for the baker's map, Commun. Math. Phys. 263 (2006) 325-352

[5] J. H. Hannay and M. V. Berry, Quantization of linear maps - Fresnel diffraction by a periodic grating, Physica D 1 (1980) 267-290

[6] B. Helffer, A. Martínez, D. Robert, Ergodicité et limite semi-classique, Commun. Math. Phys. 109 (1987), 313-326

[7] J. P. Keating, F. Mezzadri and J. M. Robbins, Quantum boundary conditions for torus maps, Nonlinearity 12 (1999) 579-591

[8] J. Marklof and S. O'Keefe, Weyl's law and quantum ergodicity for maps with divided phase space; appendix by S. Zelditch Converse quantum ergodicity, Nonlinearity 18 (2005) 277-304

[9] Z. Rudnick, The Arithmetric Theory of Quantum Maps, Proceedings of NATO Advanced Study Institute on Equidistribution in Number Theory, Montreal 2005, Nato Science Series 237, Springer, 2007 


\section{Reverse quantum ergodicity HaRUYa Mizutani}

Let $M$ be a compact Riemannian manifold (without boundary) and $\left\{\varphi_{j}\right\}_{j=1}^{\infty}$ an orthonormal basis consisting of eigenfunctions of $\sqrt{\Delta}$ with eigenvalues $\lambda_{j}$ :

$$
\sqrt{\Delta} \varphi_{j}=\lambda_{j} \varphi_{j}, 0 \leq \lambda_{1} \leq \lambda_{2} \leq \cdots .
$$

It is widely known as the quantum ergodicity theorem that if the geodesic flow on the unit cotangent bundle $S^{*} M$ is ergodic with respect to the Liouville measure $\mu$, then there exists a subsequence of full density $\left\{j_{k}\right\}_{k=1}^{\infty}$ such that for any pseudodifferential operator $A$ of order zero, we have

$$
\lim _{k \rightarrow \infty}\left\langle A \varphi_{j_{k}}, \varphi_{j_{k}}\right\rangle=\frac{1}{\mu\left(S^{*} M\right)} \int_{S^{*} M} \sigma(A) d \mu,
$$

where $\sigma(A)$ is the principal symbol of $A$. This remarkable result was established by Shnirelman, Zelditch and Colin de Verdière. The reverse implication does not holds in general and there exist counterexmaples (see, e.g., Gutkin's counterexample on two dimensional billiards [1]). However, if we assume an additional condition on the off-diagonal matrix elements or the quantum transition amplitudes $\left\langle A \varphi_{j}, \varphi_{k}\right\rangle$ with $\lambda_{j} \neq \lambda_{k}$, then we have an equivalence between the classical ergodicity and the quantum ergodicity plus such a condition. In this talk we give the proof of such an equivalence. The main theorem is the following:

Theorem 1 (Sunada [2]). Let $N(\lambda):=\sharp\left\{j ; \lambda_{j} \leq \lambda\right\}$ be the spectral counting function. The geodesic flow on $S^{*} M$ is ergodic if and only if for every pseudodifferential operator $A$ of order zero and every orthonormal basis $\left\{\varphi_{j}\right\}_{j=1}^{\infty}$ consisting of eigenfunctions of $\sqrt{\Delta}$ with eigenvalues $\lambda_{j}$, the following two conditions hold: (a)

$$
\lim _{\lambda \rightarrow+\infty} \frac{1}{N(\lambda)} \sum_{j, k, \lambda_{j}=\lambda_{k} \leq \lambda}\left|\left\langle A \varphi_{j}, \varphi_{k}\right\rangle\right|^{2}=\left|\frac{1}{\mu\left(S^{*} M\right)} \int_{S^{*} M} \sigma(A) d \mu\right|^{2} .
$$

$$
\lim _{\delta \rightarrow 0} \limsup _{\lambda \rightarrow+\infty} \frac{1}{N(\lambda)} \sum_{j, k, \lambda_{j} \leq \lambda, 0<\left|\lambda_{j}-\lambda_{k}\right|<\delta}\left|\left\langle A \varphi_{j}, \varphi_{k}\right\rangle\right|^{2}=0 .
$$

Let $\Psi^{0}$ be the space of pseudodifferential operators of order zero and $\bar{A}$ the weak limit of the time average of $A \in \Psi^{0}$ :

$$
\bar{A}:=\lim _{T \rightarrow+\infty} A_{T}, A_{T}:=\frac{1}{2 T} \int_{-T}^{T} U_{t}^{*} A U_{t} d t,
$$

where $U_{t}=e^{i t \sqrt{\Delta}}$ is the wave group. Let $\langle A\rangle$ be the space average of $A$ :

$$
\langle A\rangle:=\lim _{\lambda \rightarrow+\infty} \frac{1}{N(\lambda)} \sum_{j, \lambda_{j} \leq \lambda}\left\langle A \varphi_{j}, \varphi_{j}\right\rangle=\frac{1}{\mu\left(S^{*} M\right)} \int_{S^{*} M} \sigma(A) d \mu .
$$

The second equality follows from the local Weyl law. 
The key step of proof of Theorem 1 is the following characterization of the classical ergodicity and the reformulation of Theorem 1 in terms of the space and time averages.

Lemma 1. The followings are equivalent.

(1) The geodesic flow is ergodic.

(2) For any $A \in \Psi^{0}, \lim _{T \rightarrow+\infty}\left\langle A_{T}^{*} A_{T}\right\rangle=\left\langle\bar{A}^{*} \bar{A}\right\rangle$.

Lemma 2. Let $A \in \Psi^{0}$.

(1) The condition (a) in Theorem 1 holds if and only if

$$
\left\langle\bar{A}^{*} \bar{A}\right\rangle=|\langle A\rangle|^{2} \text {. }
$$

(2) The condition (b) in Theorem 1 holds if and only if

$$
\lim _{T \rightarrow+\infty}\left\langle A_{T}^{*} A_{T}\right\rangle=\left\langle\bar{A}^{*} \bar{A}\right\rangle
$$

We also study a quantum analogue of the classical weak mixing property. We first recall the notion of the quantum weak mixing property for $e^{i t \sqrt{\Delta}}$, which was introduced by Zelditch [3], and prove that if the geodesic flow is weak mixing, then $e^{i t \sqrt{\Delta}}$ is quantum weak mixing. We also prove the converse theorem under an additional condition on the quantum transition amplitudes:

Theorem 2 (Zelditch [3]). The geodesic flow on $S^{*} M$ is weak mixing if and only if for every $A \in \Psi^{0}$ and every orthonormal basis $\left\{\varphi_{j}\right\}_{j=1}^{\infty}$ as above, the following two conditions hold:

(a) For all $\tau \in \mathbb{R}$,

$$
\lim _{\lambda \rightarrow+\infty} \frac{1}{N(\lambda)} \sum_{j, k, \lambda_{j}=\lambda_{k}+\tau \leq \lambda}\left|\left\langle A \varphi_{j}, \varphi_{k}\right\rangle\right|^{2}=\left|\frac{1}{\mu\left(S^{*} M\right)} \int_{S^{*} M} \sigma(A) d \mu\right|^{2} \delta_{\tau 0},
$$

where $\delta_{\tau 0}=1$ if $\tau=0$ and $\delta_{\tau 0}=0$ if $\tau \neq 0$.

(b) For all $\tau \in \mathbb{R}$,

$$
\lim _{\delta \rightarrow 0} \limsup _{\lambda \rightarrow+\infty} \frac{1}{N(\lambda)} \sum_{j, k, \lambda_{j} \leq \lambda, 0<\left|\lambda_{j}-\lambda_{k}-\tau\right|<\delta}\left|\left\langle A \varphi_{j}, \varphi_{k}\right\rangle\right|^{2}=0 .
$$

\section{REFERENCES}

[1] B. Gutkin, Note on converse quantum ergodicity, Proc. Amer. Math. Soc. 137 (2009), 27952800.

[2] T. Sunada, Quantum ergodicity, Progress in inverse spectral geometry, 175-96, Trends Math., Birkhäuser, Basel, 1997.

[3] S. Zelditch, Quantum mixing, J. Funct. Analysis 140 (1996), 68-86. 


\section{Quantum ergodicity for quantum graphs Peter Gmeiner}

To define a quantum graph we start with a metric graph $G=(V, B)$, consisting of a finite set of vertices $V$ and a finite set of bonds $B$. A Schrödinger operator is defined on each bond of the metric graph and we can formulate an eigenproblem on each bond. Two boundary conditions are imposed to make this operator self-adjoint (namely continuity of the wave function on the vertices and current conservation on each vertex). Therefore we get an unbounded, discrete, non-negative spectrum. Instead of this self-adjoint extension approach [Kot99, Gnu06, Kuc04, Gnu10], there is another way to define a quantum graph which is called scattering approach. In this one considers a wave propagation on the metric graph where each vertex is treated as a scatterer and the propagation along the bonds is free. The scattering behavior at the vertices is described with a scattering matrix $S$ and the propagation along a bond with a propagation matrix. Together these unitary matrices define a quantum map which is the time evolution of the wave function on the metric graph. A metric graph $G$ together with a scattering matrix $S$ describes a quantum graph. The classical counterpart of a quantum graph is a Markov process on the graph $G$ [Gnu10].

For defining quantum ergodicity on quantum graphs we introduce observables $O$ and their quantised counterparts. Quantum ergodicity on a (finite) quantum graph is defined as follows: for every classical observable $O$ there exists a density 1 subsequence of eigenstates such that the high-energy limit of the normalized mean value of the quantization of $O$ equals the normalized mean value of the classical observable $O$. It turns out that in many cases this definition of quantum ergodicity cannot be fulfilled. To achieve a reasonable definition of quantum ergodicity we consider a sequence of quantum graphs $\left(\left(G_{l}, S_{l}\right)\right)_{l \in \mathbb{N}}$ with an increasing number of bonds $\left|B_{l}\right|<\left|B_{l+1}\right|$ and an uniform lower and upper bound on the bond lengths. Furthermore one defines an acceptable sequence of observables $\left(O_{l}\right)_{l \in \mathbb{N}}$ in the sense that the limit of their mean-values exists for $l \rightarrow \infty$ and each bond-component of $O_{l}$ is bounded from below and above. For each $l$ we consider the quantum ergodicity as defined above and take the limit $l \rightarrow \infty$ and get the notion of asymptotic quantum ergodicity on quantum graphs. The limit $l \rightarrow \infty$ plays the role of the semiclassical limit for quantum graphs. Roughly speaking a family of quantum graphs is quantum ergodic if (most of) the eigenfunctions become equidistributed in the semiclassical limit [Gnu10]

The question is now what structure the quantum graphs should share so that they are asymptotic quantum ergodic (AQE). The answer is not clear at all. For star graphs (graphs which consists of $\alpha v$ vertices which are connected only to one central vertex) it turns out that AQE cannot hold. With a specific observable which is the indicator function of the first $v$ bonds of the graph and with a direct calculation it can be proved that these family of quantum graphs is not asymptotic quantum ergodic [Ber04]. On the other hand in [Ber07] asymptotic quantum ergodicity is proved for a specific construction of graphs related to interval maps. Contrary to the usual way to prove QE in this proof the Egorov property is not 
used. Instead of this a kind of trace formula expansion of the variance between quantum mean and classical mean is used. Further one can show that it is enough to consider only diagonal terms of this expansion to prove AQE. Together with the assumed ergodicity of the interval map this variance tends to zero which is equivalent to AQE. However an Egorov property for such quantum graphs constructed from interval maps can also be deduced [Ber07].

There also exists a heuristic approach treated in [Gnu10, Gnu08] which conjectures criteria for quantum ergodicity only depending on the corresponding classical map of the quantum graph. To be more precise if one assumes that the classical spectral gap decays like $|B|^{-\alpha}$ for $\alpha \geq 0$, then one can formulate criterias for asymptotic quantum ergodicity depending only on $\alpha$ and the eigenvalues of the classical map. For the star graphs it can be verified that these conjectures hold. However a rigorous proof is missing.

A different point of view is to consider for large $d$-regular graphs the eigenstates of the discrete Laplacian. In [Elo08] it is calculated numerically that the distribution of the eigenstates coincide asymptotically with a Gaussian distribution. On the other hand in [Bro09] a delocalization theorem is proved for eigenfunctions on $d$-regular graphs satisfying a specific girth-condition (these graphs have locally the structure of a regular tree).

\section{REFERENCES}

[Ber04] G. Berkolaiko, J. P. Keating, B. Winn: No Quantum Ergodicity for Star Graphs, Communications in Mathematical Physics 250, 259 (2004).

[Ber07] G. Berkolaiko, J. P. Keating, U. Smilansky: Quantum Ergodicity for Graphs Related to Interval Maps, Communications in Mathematical Physics 273, 137 (2006).

[Bro09] S. Brooks, E. Lindenstrauss: Non-Localization of Eigenfunctions on Large regular graphs, arXiv 0912.3239v1, (2009).

[Elo08] Y. ELON: Eigenvectors of the discrete Laplacian on regular graphs - a statistical approach, Journal of Phys. A 41, (2008).

[Gnu06] S. Gnutzmann, U. Smilansky: Quantum graphs: Application to quantum chaos and universal spectral statistics, Advances in Physics 55, 527 (2006).

[Gnu08] S. Gnutzmann, J.P. Keating, F. Piotet: Quantum Ergodicity on Graphs, Physical Review Letters 101, (2008).

[Gnu10] S. Gnutzmann, J.P. Keating, F. Piotet: Eigenfunction Statistics on Quantum Graphs, Annals of Physics 325, 2595 (2010).

[Kot99] T. Kottos, U. Smilansky: Periodic Orbit Theory and Spectral Statistics for Quantum Graphs, Annals of Physics 274, 1, 76 (1999).

[Kuc04] P. Kuchment: Quantum graphs I. Some Basic Structures, Waves Random Media 14, 107 (2004).

\section{Quantum Ergodicity for Systems Sebastian Goette}

In this talk, we investigate quantum ergodicity in the high energy limit for geometric Dirac and Laplace operators acting on sections of vector bundles over a compact Riemannian manifold $(M, g)$. We follow the approach of Jakobson and Strohmaier in [3]. 
For the examples below, it is not sufficient to require ergodicity of the geodesic flow of $(M, g)$ acting on the unit cotangent bundle $\pi: T_{1}^{*} M \rightarrow M$. Instead, one considers the geodesic frame flow. It is defined on the bundle $F \rightarrow M$ of $g$-orthonormal (co-)frames in $T^{*} M$. The map $\left(\xi^{1}, \ldots, \xi^{n}\right) \mapsto \xi^{1} \in T_{1}^{*} M$ turns $F$ into an $O(n-1)$-principal bundle over $T_{1}^{*} M$. The frame flow on $F$ translates $\left(\xi^{1}, \ldots, \xi^{n}\right)$ parallelly along the geodesic in $T_{1}^{*} M$ with start (co-)vector $\xi^{1}$. The frame flow is known to be ergodic for all smooth metrics that are sufficiently close to a metric of constant negative curvature, and for an open and dense subset of the space of negatively curved metrics, see [1] for an overview of known results. It is conjectured to be ergodic whenever the manifold is strictly $\frac{1}{4}$-pinched, that is, if all sectional curvatures lie in the interval $\left[-1,-\frac{1}{4}\right)$.

Next, let $P$ be an elliptic classical pseudodifferential operator acting on a vector bundle $E \rightarrow M$ with scalar principal symbol $\sigma_{P}(\xi)=1$ for all $\xi \in T_{1}^{*} M$. Then the subprincipal symbol sub $P$ of Duistermaat and Hörmander [2] induces a partial connection on the pullback $\pi^{*} E$ to $T_{1}^{*} M$ in the direction of the geodesic flow. For example, if $\Delta$ is a Laplacian associated to a connection $\nabla^{E}$, then sub $\Delta$ gives the partial connection induces by the pullback of $\nabla^{E}$. This way, the operator $P$ defines a well-defined lift of the geodesic flow to a flow on $\pi^{*} E$. It also induces a flow on $C^{\infty}\left(T_{1}^{*} M ; \pi^{*}\right.$ End $\left.E\right)$.

Theorem 1 (Egorov's Theorem for Laplace Operators, [3, Theorem 1.4]). If $A$ is a classical pseudodifferential operator of degree 0 on $E \rightarrow M$ and $P$ is as above and of degree 1 , then for all $t \in \mathbb{R}$, the operators $A_{t}=e^{i t P} A e^{-i t P}$ are again classical pseudodifferential operator of degree 0 with

$$
\sigma_{A_{t}}=\beta^{t}\left(\sigma_{A}\right) \quad \in \quad C^{\infty}\left(T_{1}^{*} M ; \pi^{*} \text { End } E\right) .
$$

Recall that a state of a $C^{*}$-algebra $\mathcal{A}$ is a positive linear functional $\omega \in \mathcal{A}^{*}$ with $\omega(1)=1$. Here, states of $\mathcal{A}=C\left(T_{1}^{*} M ; \pi^{*}\right.$ End $\left.E\right)$ play the role of probability measures on $T_{1}^{*} M$ in the other talks. In particular, the flow $\beta^{t}$ acts on the states of $\mathcal{A}$, and the invariant states of $\beta^{t}$ form a convex set $E_{\mathcal{A}}^{\beta^{t}}$. Its extremal points are called ergodic states. Each invariant state can be decomposed as a sum or integral of ergodic states, but in contrast to the commutative situation, this decomposition is in general far from unique.

As an example, consider the Dirac operator $D$ acting on spinors, that is sections of the spinor bundle $S \rightarrow M$. Its symbol is given by Clifford multiplication $\gamma$. For $\xi \in T_{1}^{*} M$, Clifford multiplication $\gamma_{\xi}$ has eigenvalues \pm 1 , so we obtain a splitting of $\pi^{*} S$ into two bundles of eigenvectors of the same rank, corresponding roughly to the eigenspaces of the operator $\operatorname{sign}(D)$. Let $P_{ \pm} \in$ End $\pi^{*} S$ denote the corresponding projections, and let $\mu$ denote the normalized Liouville measure on $T_{1}^{*}(M)$. The following result is one of the central steps towards quantum ergodicity.

Theorem 2 ([3, Theorem 4.3]). If the frame flow is ergodic, then the states

$$
\omega_{ \pm}: a \longmapsto \frac{2}{\operatorname{rk} S} \int_{T_{1}^{*} M} \operatorname{tr}\left(P_{ \pm, \xi} \circ a_{\xi} \circ P_{ \pm, \xi}\right) d \mu(\xi)
$$

are ergodic with respect to the flow $\beta^{t}$ on $\mathcal{A}$. 
In particular, the normalized trace $\omega=\frac{1}{2}\left(\omega_{+}+\omega_{-}\right)$on $\mathcal{A}=C\left(T_{1}^{*} M ; \pi^{*}\right.$ End $\left.S\right)$, which would be the natural generalisation of the Liouville measure, is not $\beta^{t}$ ergodic. If $M$ is even-dimensional, then the classical splitting $S=S^{+} \oplus S^{-}$gives another ergodic decomposition of $\omega$.

Let now $\left(\varphi_{k}\right)_{k \in \mathbb{N}}$ be an orthonormal sequence of eigenspinors for eigenvalues $0 \leq$ $\lambda_{k} \nearrow \infty$ that spans $\frac{1+\operatorname{sign}(D)}{2} L^{2}(M ; S)$. Then each $\varphi_{k}$ defines a state on the $C^{*}-$ algebra $\mathcal{B}$ of degree 0 classical pseudodifferential operators acting on $S$ by

$$
A \mapsto\left\langle\varphi_{k}, A \varphi_{k}\right\rangle .
$$

Theorem 3 ([3, Theorem 4.4]). If the frame flow is ergodic, then quantum ergodicity holds, i.e.

$$
\lim _{N \rightarrow \infty} \frac{1}{N} \sum_{k=1}^{N}\left|\left\langle\varphi_{k}, A \varphi_{k}\right\rangle-\omega_{+}\left(\sigma_{A}\right)\right|=0 \quad \text { for all } A \in \mathcal{B} .
$$

Moreover, there exists a density one subsequence $\varphi_{k}^{\prime}$ such that

$$
\lim _{k \rightarrow \infty}\left\langle\varphi_{k}^{\prime}, A \varphi_{k}^{\prime}\right\rangle=\omega_{+}\left(\sigma_{A}\right) \quad \text { for all } A \in \mathcal{B} .
$$

Similar statements hold for the negative eigenspinors if $\omega_{+}$is replaced by $\omega_{-}$. Jakobson and Strohmaier also prove a related version of quantum ergodicity for the Hodge-Laplacian on $p$-forms.

\section{REFERENCES}

[1] K. Burns, M. Pollicott, Stable ergodicity and frame flows, Geom. Dedicata 98 (2003), 189210

[2] J.J. Duistermaat,L. Hörmander, Fourier integral operators ii, Acta Mathematica 128 (1972), 183-269

[3] D. Jakobson, A. Strohmaier, High energy limits of Laplacetype and Dirac-type eigenfunctions and frame flows, Commun. Math. Phys. 270 (2007), 813-833

\section{The rate of quantum ergodicity}

\section{HOLGER THEN}

In the semiclassical limit, the matrix elements of smooth operators tend to the microcanonical average for almost all eigenfunctions

$$
\langle n|\hat{A}| n\rangle \underset{\text { weak limit }}{\stackrel{n \rightarrow \infty}{\longrightarrow}}\{A\}:=\int d p d q \delta(E-H(p, q)) A(p, q) / \Omega .
$$

We are interested in the rate with which this limit is approached. A simple heuristic derivation for the rate was first given by Feingold and Peres [1] who inspected the variance of non-diagonal matrix elements $A_{j k}:=\langle j|\hat{A}| k\rangle$. Consider

$$
\sum_{k} e^{\frac{i}{\hbar}\left(E_{j}-E_{k}\right) t}\left|A_{j k}\right|^{2}=\sum_{k}\left\langle j\left|e^{\frac{i}{\hbar} \hat{H} t} \hat{A} e^{-\frac{i}{\hbar} \hat{H} t}\right| k\right\rangle\langle k|\hat{A}| j\rangle=\langle j|\hat{A}(t) \hat{A}(0)| j\rangle
$$


where $\hat{A}(t)$ and $\hat{A}(0)$ are given in the Heisenberg picture. In the semiclassical limit this ought to tend to the autocorrelation of the classical dynamical variable,

$$
C(t):=\{A(t) A(0)\}=\lim _{T \rightarrow \infty} \frac{1}{T} \int_{0}^{T} A(t+\tau) A(\tau) d \tau .
$$

Its Fourier transform $\int C(\omega) e^{-i \omega t} d t$ is called the power spectrum of $A$. There is a difficulty, however. The autocorrelation $C(t)$ is not yet of Schwartz class. Subtracting $A_{j j}^{2}$ from (2) and $\{A\}^{2}$ from (3) results in the regularized power spectrum

$$
S(\omega):=\int_{-\infty}^{\infty}\left(C(t)-\{A\}^{2}\right) e^{-i \omega t} d t .
$$

Approximating the sum in (2) by an integral, $\sum_{k}^{\prime} \ldots \rightarrow \int \ldots \rho\left(E_{k}\right) d E_{k}$, where

$$
\rho(E):=(2 \pi \hbar)^{-d} \int \delta(E-H(p, q)) d p d q
$$

is the course-grained density of states, allows to solve for the quantum variance

$$
\left|A_{j k}\right|^{2} \simeq S\left(\left(E_{j}-E_{k}\right) / \hbar\right) / 2 \pi \hbar \rho(E) .
$$

The limit $\omega \rightarrow 0$ relates the quantum variance to the classical variance

$$
\begin{aligned}
2 \pi \hbar \rho(E)\left|A_{j k}\right|^{2}=S(0) & =\int_{-\infty}^{\infty}\left(C(t)-\{A\}^{2}\right) d t \\
& =\lim _{T \rightarrow \infty} \frac{1}{T}\left\langle\left|\int_{0}^{T}(A(t)-\{A\}) d t\right|^{2}\right\rangle .
\end{aligned}
$$

Of course the limit implies $\left|E_{j}-E_{k}\right| \rightarrow 0$, but $j \neq k$, because the regularization has removed the diagonal terms which are no longer accessible.

Quantum variance conjecture 1. Non-diagonal matrix elements are distributed around zero with variance

$$
\left|A_{j k}\right|^{2}=\frac{S(0)}{2 \pi \hbar \rho(E)} \propto \hbar^{d-1} .
$$

With more sophisticated methods, but still heuristic, Eckhardt, Fishman, Keating et al. could solve for the quantum variance of diagonal elements [2].

Quantum variance conjecture 2. Diagonal matrix elements are Gaussian distributed around their classical mean with variance

$$
\left|A_{j j}-\{A\}\right|^{2}=g \frac{S(0)}{2 \pi \hbar \rho(E)} \propto \hbar^{d-1},
$$

where the symmetry factor $g$ takes the value 2 or 1 depending on whether the system is invariant under time-reversal or not.

The quantum variance conjecture(s) reach far beyond of what can be proven. Rigorous upper bounds on the quantum variance were obtained in [3] for geodesic flows and in [4] for Hamiltonian flows. We present the latter. Assume $M$ is either a smooth compact manifold or $M=\mathbb{R}^{d}$. Let $\mathcal{H} \in \Psi^{m}(M), m>0$, be selfadjoint with principal symbol $H_{0}$, and $\phi^{t}$ be the Hamiltonian flow generated 
by $H_{0}$. Assume $E$ is a regular value of $H_{0}$ and that the energy shell $\Sigma_{E}:=$ $\left\{(x, \xi) \in T^{*} M ; H_{0}(x, \xi)=E\right\}$ is compact. Hence, the spectrum of $\mathcal{H}$ is discrete in a neighbourhood of $E$. Denote by $E_{n}, \psi_{n}$ the eigenvalues and eigenfunctions of $\mathcal{H}$ in the interval $I(E, \hbar):=[E-\alpha \hbar, E+\alpha \hbar], \alpha>0$. If furthermore the set of periodic orbits of the Hamiltonian flow $\phi^{t}$ on $\Sigma_{E}$ has measure zero, the number of eigenvalues close to $E$ satisfy the Weyl estimate

$$
N(I(E, \hbar))=\frac{2 \alpha}{(2 \pi)^{d} \hbar^{d-1}} \operatorname{vol}\left(\Sigma_{E}\right)(1+o(1)) .
$$

For $a \in C^{\infty}\left(T^{*} M\right)$, the classical autocorrelation function is defined as

$$
C_{E}[a](t):=\frac{1}{\operatorname{vol}\left(\Sigma_{E}\right)} \int_{\Sigma_{E}} a \circ \phi^{t} a d \mu_{E}-\left(\bar{a}_{E}\right)^{2},
$$

where $d \mu_{E}$ is the Liouville measure on $\Sigma_{E}$, and $\bar{a}_{E}$ denotes the classical average,

$$
\bar{a}_{E}:=\frac{1}{\operatorname{vol}\left(\Sigma_{E}\right)} \int_{\Sigma_{E}} a d \mu_{E}
$$

We call $\phi^{t}$ to be ergodic with rate $\gamma>0$ on $\Sigma_{E}$ if for all $a \in C^{\infty}\left(\Sigma_{E}\right)$ and $f \in \mathcal{S}(\mathbb{R})$ there exists a positive constant $C$ such that

$$
\left|\frac{1}{T} \int f\left(\frac{t}{T}\right) C_{E}[a](t) d t\right| \leq C(1+|T|)^{-\gamma} .
$$

And we call $\phi^{t}$ to be weak mixing with rate $\gamma>0$ on $\Sigma_{E}$ if for all $a \in C^{\infty}\left(\Sigma_{E}\right)$ and $f \in \mathcal{S}(\mathbb{R})$ there exists a positive constant $C$ such that for all $\varepsilon \in \mathbb{R}$

$$
\left|\frac{1}{T} \int f\left(\frac{t}{T}\right) C_{E}[a](t) e^{i \varepsilon t} d t\right| \leq C(1+|T|)^{-\gamma} .
$$

For smooth bounded operators $A \in \Psi^{0}(M)$ with principal symbol $\sigma(A)$ the following theorems hold [4]:

Theorem 1. If $\phi^{t}$ is ergodic with rate $\gamma>0$ on $\Sigma_{E}$, then there exists a positive constant $C>0$ such that

(15) $\frac{1}{N(I(E, \hbar))} \sum_{E_{n} \in I(E, \hbar)}\left|\left\langle\psi_{n}, A \psi_{n}\right\rangle-\overline{\sigma(A)}\right|^{2} \leq \begin{cases}C|\log \hbar|^{-\gamma} & \text { if } 0<\gamma<1 \\ C|\log \hbar|^{-1} & \text { if } \gamma \geq 1\end{cases}$

Theorem 2. If $\phi^{t}$ is ergodic with rate $\gamma>0$ on $\Sigma_{E}$, then there exists a positive constant $C>0$ such that

$$
\frac{1}{N(I(E, \hbar))} \sum_{\substack{n, m \\ E_{n} \in I(E, \hbar) \\\left|E_{m}-E_{n}\right| \leq \hbar /|\log \hbar|}}^{\prime}\left|\left\langle\psi_{m}, A \psi_{n}\right\rangle\right|^{2} \leq \begin{cases}C|\log \hbar|^{-\gamma} & \text { if } 0<\gamma<1 \\ C|\log \hbar|^{-1} & \text { if } \gamma \geq 1\end{cases}
$$


Theorem 3. If $\phi^{t}$ is weak mixing with rate $\gamma>0$ on $\Sigma_{E}$, then there exists a positive constant $C>0$ such that for all $\varepsilon \in \mathbb{R}$

$$
\frac{1}{N(I(E, \hbar))} \sum_{\substack{n, m \\ E_{n} \in I(E, \hbar) \\\left|E_{m}-E_{n}+\hbar \varepsilon\right| \leq \hbar /|\log \hbar|}}^{\prime}\left|\left\langle\psi_{m}, A \psi_{n}\right\rangle\right|^{2} \leq \begin{cases}C|\log \hbar|^{-\gamma} & \text { if } 0<\gamma<1 \\ C|\log \hbar|^{-1} & \text { if } \gamma \geq 1\end{cases}
$$

Proof. Let $\rho$ be a smooth function on $\mathbb{R}$ such that its Fourier transform $\hat{\rho}$ has compact support in a small neighbourhood of zero which contains no period of a periodic orbit of $\Phi^{t}$ on $\Sigma_{E}$. Moreover, we choose $\rho \geq 0$, and $\rho\left(\left(E-E_{n}\right) / \hbar\right) \geq 1$ for $E_{n} \in I(E, \hbar)$. Let $f$ such that $\hat{f} \in C^{\infty}([-1,1])$ and set $f_{T}(\tau)=f(T \tau)$ so that $\widehat{f_{T}}(t)=\hat{f}(t / T) / T$. Furthermore, we choose $f_{T} \geq 0$, and $f_{T}\left(\left(E_{m}-E_{n}\right) / \hbar\right) \geq 1$ for $\left|E_{m}-E_{n}\right| \leq \hbar /|\log \hbar|$. Hence,

(18)

$$
\begin{aligned}
& \sum_{n, m}\left|\left\langle\psi_{m}, O p[a] \psi_{n}\right\rangle-\delta_{m n} \bar{a}_{E}\right|^{2} \\
& E_{n} \in I(E, \hbar) \\
& \left|E_{m}-E_{n}+\hbar \varepsilon\right| \leq \hbar /|\log \hbar| \\
& \leq \sum_{n, m} \rho\left(\frac{E-E_{n}}{\hbar}\right) f_{T}\left(\frac{E_{m}-E_{n}+\hbar \varepsilon}{\hbar}\right)\left|\left\langle\psi_{m}, O p[a] \psi_{n}\right\rangle-\delta_{m n} \bar{a}_{E}\right|^{2} \\
& =\sum_{n, m} \rho\left(\frac{E-E_{n}}{\hbar}\right) \int e^{\frac{i}{\hbar}\left(E_{m}-E_{n}\right) t} e^{i \varepsilon t} \widehat{f_{T}}(t) d t\left\langle\psi_{n},\left(O p[a]-\bar{a}_{E}\right)^{*} \psi_{m}\right\rangle\left\langle\psi_{m},\left(O p[a]-\bar{a}_{E}\right) \psi_{n}\right\rangle
\end{aligned}
$$

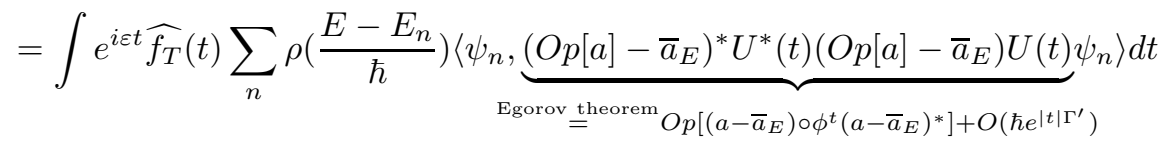

$$
\begin{aligned}
& \left.\stackrel{\text { local Weyl law } \int e^{i \varepsilon t} \widehat{f_{T}}(t)(\frac{\hat{\rho}(0)}{(2 \pi)^{d} \hbar^{d-1}} \underbrace{\overline{\sigma\left(\left(a-\bar{a}_{E}\right) \circ \phi^{t}\left(a-\bar{a}_{E}\right)\right)}}_{=C_{E}[\sigma(a)](t)} E}{=}+O\left(\hbar^{d-2} e^{|t| \Gamma}\right)\right) d t \\
& =\frac{\hat{\rho}(0)}{(2 \pi)^{d} \hbar^{d-1}}(\underbrace{\int \widehat{f_{T}}(t) C_{E}[\sigma(a)](t) e^{i \varepsilon t} d t}_{|\cdot| \leq C(1+|T|)^{-\gamma}}+O\left(\hbar|\hat{f}|_{0} \frac{1}{\Gamma T} e^{\Gamma T}\right)) \\
& T:=\frac{1}{\Gamma} \stackrel{\log \hbar \mid}{=} \hbar^{1-d}\left(O\left(|\log \hbar|^{-\gamma}\right)+O\left(|\log \hbar|^{-1}\right)\right)
\end{aligned}
$$

(18) is an upper bound on the sum of diagonal and non-diagonal elements. Dividing by $N(I(E, \hbar))$ this proves theorem 3 , and the special choice $\varepsilon=0$ yields theorems 1 and 2 .

As shown in [5], the upper bound is optimal if no further assumptions are made. In particular, explicit examples of quantum maps can be constructed for which the logarithmic upper bound is sharp.

\section{REFERENCES}

[1] M. Feingold and A. Peres, Phys. Rev. A 34 (1986) 591-595. 
[2] B. Eckhardt, S. Fishman, J. Keating, O. Agam, J. Main, and K. Müller, Phys. Rev. E 52 (1995) 5893-5903.

[3] S. Zelditch, Commun. Math. Phys. 160 (1994) 81-92.

[4] R. Schubert, Ann. Henri Poincaré 7 (2006) 1085-1098.

[5] R. Schubert, Ann. Henri Poincaré 9 (2008) 1455-1477.

\section{Arithmetic Quantum Unique Ergodicity LIOR ROSENZWEIG}

Let $M$ be a compact Riemannian manifold of negative curvature. Then by the Quantum Ergodicity Theorem (QET, $[5,6,2])$, if $\left\{\phi_{j}(x)\right\}$ is a sequence of normalized eigenfunctions of the Laplacian $\Delta_{M}$ on $M$, then there exists a subset $\Lambda \subset \mathbb{N}$ of density 1 , such that

$$
\mu_{j}:=\left|\phi_{j}\right|^{2} \operatorname{dvol}_{M} \rightarrow \operatorname{dvol}_{M} \quad \text { as } j \rightarrow \infty, j \in \Lambda .
$$

The Quantum Unique Ergodicity conjecture, raised by Rudnick and Sarnak in [4] states that in fact in this case $\Lambda=\mathbb{N}$, that is there are no exceptional quantum limits. A special case of this conjecture, is the case where $M=\Gamma \backslash \mathbb{H}$, where

$$
\mathbb{H}:=\{z \in \mathbb{C}: \operatorname{Im}(z)>0\}
$$

is the upper half plane equipped with the hyperbolic metric, and $\Gamma$ is of arithmetic nature. In this case the arithmetic structure of the system can be taken into consideration. In particular, the Laplacian operator $\Delta$ comes with natural arithmetic commuting symmetries called "Hecke operators". When considering joint eigenfunctions of the Laplacian and of all Hecke operators, extra information is given on the eigenfunction and the behaviour of $\mu_{j}$. Such quantum limits are called arithmetic quantum limits. For example, in [1] Bourgain and Lindenstrauss proved the following Theorem

Theorem 1. Let $M=\Gamma \backslash \mathbb{H}$ be such that $\Gamma$ is a lattice in $S L_{2}(\mathbb{R})$ corresponding to the norm one elements of an order in a $\mathbb{R}$-split quaternion algebra, defined over $\mathbb{Q}$. Then there exists $\kappa>0$, such that for any arithmetic quantum limit $\mu$, almost every ergodic component has entropy $\geq \kappa$.

In [3] Lindenstrauss proved the following theorem

Theorem 2. Let $M=\Gamma \backslash \mathbb{H}$ with $\Gamma$ a congruence lattice over $\mathbb{Q}$. Then if $M$ is compact the only quantum limit is the normalized volume dvol v. $_{\text {. }}$.

The proof of Theorem 2 is based on Theorem 1 and on a measure rigidity result proved in [3].

In the talk we will go over the proof of Theorem 2 and Theorem 1 . In particular we will define the notion of quaternion algebras, orders and lattices coming from the norm one elements of quaternion algebras, and define the Hecke operators explicitly in this case. We will later follow the proof given in [1] to get the positive entropy result. If time permits a sketch of the proof of Theorem 2 using the measure rigidity theorem will be described. 


\section{REFERENCES}

[1] J. Bourgain and E. Lindenstrauss, Entropy of quantum limits Comm. Math. Phys., 233(1):153-171, 2003.

[2] Y. Colin de Verdière, Ergodicité et fonctions propres du laplacien Comm. Math. Phys., 102(3):497-502, 1985.

[3] E. Lindenstrauss, Invariant measures and arithmetic quantum unique ergodicity Ann. of Math. (2), 163(1):165-219, 2006.

[4] Z. Rudnick and Peter Sarnak. The behaviour of eigenstates of arithmetic hyperbolic manifolds Comm. Math. Phys., 161(1):195-213, 1994.

[5] A. I. Shnirelman. Ergodic properties of eigenfunctions Uspehi Mat. Nauk, 29(6(180)):181$182,1974$.

[6] S. Zelditch. Uniform distribution of eigenfunctions on compact hyperbolic surfaces Duke Math J., 55(4):919-941, 1987.

\section{QUE for the modular surface (after Luo and Sarnak) NiCOLE RAULF}

In this talk we discussed the quantum unique ergodicity result of Luo and Sarnak [3] for the Eisenstein series $E(z, 1 / 2+i t)$ and their proof of this result. The main references for this talk are [3] and [1], [2] for the necessary background. Let $\mathbb{H}^{2}:=\{z \in \mathbb{C}: \operatorname{Im} z>0\}$ be the upper half-plane equipped with the hyperbolic metric. The corresponding volume element is denoted by $d \mu(z)=\frac{d x d y}{y^{2}}$ and the corresponding Laplace-Beltrami operator is

$$
\Delta=y^{2}\left(\frac{\partial^{2}}{\partial x^{2}}+\frac{\partial^{2}}{\partial y^{2}}\right) .
$$

If $\Gamma_{\infty}:=\{\gamma \in \Gamma: \gamma \infty=\infty\}$ denotes the stabilizer of the cusp $\infty$ of $\Gamma:=\operatorname{PSL}_{2}(\mathbb{Z})$, the Eisenstein series $E(z, s)$ is given by

$$
E(z, s)=\sum_{\gamma \in \Gamma_{\infty} \backslash \Gamma}(\operatorname{Im}(\gamma z))^{s},
$$

$\operatorname{Re}(s)>1$. Using its Fourier expansion one sees that the Eisenstein series can be meromorphically continued to the whole complex $s$-plane. The Eisenstein series satisfies the eigenvalue equation $-\Delta E(z, s)=s(1-s) E(z, s)$ and the continuous part of the spectrum of $-\Delta$ on $L^{2}\left(\Gamma \backslash \mathbb{H}^{2}\right)=\left\{f: \mathbb{H}^{2} \rightarrow \mathbb{C}: f(\gamma z)=f(z) \forall \gamma \in \Gamma\right.$, $\left.\int_{\Gamma \backslash \mathbb{H}^{2}}|f|^{2} d \mu<\infty\right\}$ can be given with the help of the Eisenstein series $E(z, 1 / 2+i t)$, $t \geq 0$ (cf. e.g. [2]). However, $E(z, s)$ is not square integrable. Luo and Sarnak proved the following quantum unique ergodicity theorem for the generalized eigenfunctions $E(z, 1 / 2+i t)$ of $-\Delta$ :

Theorem 1 ([3]). Let $A, B$ be compact Jordan measurable subsets of $\Gamma \backslash \mathbb{H}^{2}$, then

$$
\lim _{t \rightarrow \infty} \frac{\int_{A}|E(z, 1 / 2+i t)|^{2} d \mu(z)}{\int_{B}|E(z, 1 / 2+i t)|^{2} d \mu(z)}=\frac{\mu(A)}{\mu(B)} .
$$

The proof of this theorem uses the spectral decomposition of $L^{2}\left(\Gamma \backslash \mathbb{H}^{2}\right)$, subconvexity bounds for $L$-series and properties of Hecke operators. 
Definition 1. (a) We call a smooth function $f: \mathbb{H}^{2} \rightarrow \mathbb{C}$ a Maaß form for $\Gamma$ if

(1) $f(\gamma z)=f(z) \forall \gamma \in \Gamma$,

(2) $-\Delta f=\lambda f$,

(3) $f(x+i y)=O\left(y^{N}\right)$ as $y \rightarrow \infty$ for some $N \in \mathbb{N}$.

(b) If $f$ is a Maaß form and $\int_{0}^{1} f(z) d x=0$, then $f$ is called a Maaß cusp form.

Definition 2. Let $h$ be a smooth function of compact support on $(0 ; \infty)$. Then

$$
F_{h}(z)=\sum_{\gamma \in \Gamma_{\infty} \backslash \Gamma} h(\operatorname{Im}(\gamma z))
$$

is called an incomplete Eisenstein series.

$L^{2}\left(\Gamma \backslash \mathbb{H}^{2}\right)$ can now be decomposed as follows:

Theorem 2. Let $\mathcal{C}\left(\Gamma \backslash \mathbb{H}^{2}\right)$ be the space spanned by cusp forms and $\mathcal{E}\left(\Gamma \backslash \mathbb{H}^{2}\right)$ be the space of incomplete Eisenstein series. Then

$$
L^{2}\left(\Gamma \backslash \mathbb{H}^{2}\right)=\mathcal{C}\left(\Gamma \backslash \mathbb{H}^{2}\right) \oplus \mathcal{E}\left(\Gamma \backslash \mathbb{H}^{2}\right) .
$$

Proof. See [2].

Apart from this decomposition Hecke operators play an important role in the proof of Theorem 1.

Definition 3. For $n \in \mathbb{N}$ let $\mathcal{M}(n):=\left\{\left(\begin{array}{ll}a & b \\ c & d\end{array}\right): a, b, c, d \in \mathbb{Z}, a d-b c=n\right\}$. Then for a $\Gamma$-invariant function $f: \mathbb{H}^{2} \rightarrow \mathbb{C}$ the Hecke operator $T_{n}$ is defined by

$$
T_{n} f(z)=\frac{1}{\sqrt{n}} \sum_{\gamma \in \mathrm{SL}_{2}(\mathbb{Z}) \backslash \mathcal{M}(n)} f(\gamma z) .
$$

The Hecke operators are self-adjoint in $L^{2}\left(\Gamma \backslash \mathbb{H}^{2}\right)$, they commute and they commute with the Laplace operator. Therefore we can choose a system of eigenfunctions of $-\Delta$ that are also eigenfunctions of each Hecke operator (cf. e.g. [2]).

From the recurrence relation of the Hecke operators as well as from subconvexity bounds for the L-function of a Maaß cusp form we infer

Proposition 1 ([3]). Let $\varphi_{j}$ be a Hecke Maaß cusp form. Then

$$
I_{j}(t)=\int_{\Gamma \backslash \mathbb{H}^{2}} \varphi_{j}(z)\left|E\left(z, \frac{1}{2}+i t\right)\right|^{2} d \mu(z) \ll|t|^{-1 / 6+\epsilon}
$$

for all $\epsilon>0$ where the implied constant depends on $\epsilon$ and $j$.

Moreover, using again subconvexity bounds and the explicit form of the scattering matrix for $\Gamma$ we get

Proposition 2 ([3]). Let $h \in C^{\infty}\left(\mathbb{R}^{+}\right)$be rapidly decreasing at 0 and $\infty$ and $F_{h}(z)=\sum_{\gamma \in \Gamma_{\infty} \backslash \Gamma} h(\operatorname{Im}(\gamma z))$. Then there exists a constant $c>0$ such that

$$
\int_{\Gamma \backslash \mathbb{H}^{2}} F_{h}(z)\left|E\left(z, \frac{1}{2}+i t\right)\right|^{2} d \mu(z) \sim c\left(\int_{\Gamma \backslash \mathbb{H}^{2}} F_{h}(z) d \mu(z)\right) \log t \quad \text { as } t \rightarrow \infty .
$$


Proposition 1 and Proposition 2 and an approximation argument finally yield that

$$
\int_{A}\left|E\left(z, \frac{1}{2}+i t\right)\right|^{2} d \mu(z) \sim c \mu(A) \log t
$$

as $t \rightarrow \infty$ which implies Theorem 1 .

\section{REFERENCES}

[1] D. Bump, Automorphic Forms and Representations, Cambridge Studies in Advanced Mathematics 55, Cambridge University Press, Cambridge (1998).

[2] H. Iwaniec, Introduction to the Spectral Theory of Automorphic Forms, Biblioteca de la Revista Matemática Iberoamericana, Revista Matemática Iberoamericana, Madrid (1995).

[3] W. Luo, P. Sarnak, Quantum ergodicity of eigenfunctions on $\mathrm{PSL}_{2}(\mathbb{Z}) \backslash \mathbb{H}^{2}$, Inst. Hautes Études Sci. Publ. Math. 81 (1995), 207-237.

\section{Quantum unique ergodicity for the quantised toral automorphisms BRIAN WINN}

A quantisation procedure for a linear map $\kappa$ of the 2-torus $\mathbb{T}^{2}$ was introduced by Hannay and Berry [1] (see the contribution of Karsten Fritzsch in this volume). The procedure assigns an $N$-dimensional Hilbert space of states $\mathcal{H}_{N}$, where $N=$ $1 / h$ is the inverse Planck constant; to every smooth observable $f \in C^{\infty}\left(\mathbb{T}^{2}\right)$ a Hermitian operator $\mathrm{Op}_{N}^{\mathrm{W}}(f)$ on $\mathcal{H}_{N}$; and a quantum map $U_{N}(\kappa)$ which is a unitary $N \times N$ matrix. To avoid discussion of so-called quantum boundary conditions we will restrict attention to Anosov maps $\kappa$ from the group

$$
\Gamma_{\theta}=\left\{\left(\begin{array}{ll}
a & b \\
c & d
\end{array}\right) \in \mathrm{SL}_{2}(\mathbb{Z}): a b \equiv d c \equiv 0 \quad \bmod 2\right\} .
$$

Kurlberg and Rudnick [3] outline a quantisation $U_{N}(\cdot)$ of maps that by construction depends only on the reduction of $\kappa$ modulo $2 N$ (a difference from the HannayBerry construction) and prove that if one restricts further to the subgroups

$$
\Gamma(4,2 N)=\left\{g \in \mathrm{SL}_{2}\left(\frac{\mathbb{Z}}{2 N \mathbb{Z}}\right):\left\{\begin{array}{lll}
g \equiv I & \bmod 4, & (N \text { even }) \\
g \equiv I & \bmod 2, & (N \text { odd })
\end{array}\right\}\right.
$$

then the quantisation preserves commutativity in the sense that if

$$
\kappa_{1} \kappa_{2} \equiv \kappa_{2} \kappa_{1} \quad \bmod 2 N
$$

for $\kappa_{1}, \kappa_{2} \in \Gamma(4,2 N)$, then

$$
U_{N}\left(\kappa_{1}\right) U_{N}\left(\kappa_{2}\right)=U_{N}\left(\kappa_{2}\right) U_{N}\left(\kappa_{1}\right) .
$$

It is a consequence of the linearity of the torus maps that the quantum maps obey an exact version of the Egorov property: if $T_{N}(n)$ is the quantisation of the smooth observable $e(n x)=\mathrm{e}^{2 \pi \mathrm{i} n x}, n \in \mathbb{Z}^{2}, x \in \mathbb{T}^{2}$, then

$$
U_{N}(\kappa)^{-1} T_{N}(n) U_{N}(\kappa)=T_{N}(n \kappa) .
$$


Let $\left\{\phi_{j}\right\}_{j=1}^{N}$ be an orthonormal basis of eigenvectors of $U_{N}(\kappa)$. We say that quantum unique ergodicity holds if for any $f \in C^{\infty}\left(\mathbb{T}^{2}\right)$ and any sequence $\left(j_{N}\right)_{N \rightarrow \infty}$, $j_{N} \in\{1, \ldots, N\}$ we have

$$
\lim _{N \rightarrow \infty}\left\langle\mathrm{Op}_{N}^{\mathrm{W}}(f) \phi_{j_{N}}, \phi_{j_{N}}\right\rangle=\int_{\mathbb{T}^{2}} f \mathrm{~d} x .
$$

The following criterion for quantum unique ergodicity is proved in [3]:

Proposition 1. If $\left\{\phi_{j}\right\}_{j=1}^{N}$ is an orthonormal basis of eigenvectors of $U_{N}(\kappa)$ and if for any $n \in \mathbb{Z}^{2} \backslash\{(0,0)\}$ and some $\omega$ independent of $n$ and $N$,

$$
\sum_{j=1}^{N}\left|\left\langle T_{N}(n) \phi_{j}, \phi_{j}\right\rangle\right|^{4} \leq|n|^{\omega} \mathrm{O}(1) \quad \text { as } N \rightarrow \infty,
$$

then quantum unique ergodicity holds.

In [3] Kurlberg and Rudnick construct an Abelian subgroup of unitary matrices commuting with $U_{N}(\kappa)$ and call a joint basis of eigenfunctions of $U_{N}(\kappa)$ and this group a Hecke basis. They prove that a Hecke basis eigenfunctions exhibits quantum unique ergodicity.

We briefly summarize the construction, which is based on algebraic number theory. Denote by $\alpha$ the largest eigenvalue of $\kappa$. Then $\alpha$ is a unit in the quadratic field $K=\mathbb{Q}(\alpha)$. Let $\mathfrak{O}=\mathbb{Z}[\alpha]$. Let $\left(v_{1}, v_{2}\right) \in \mathfrak{O}^{2}$ be the corresponding eigenvector to $\alpha$ and define

$$
I=\left\{n_{1} v_{1}+n_{2} v_{2}: n \in \mathbb{Z}^{2}\right\} .
$$

Then by construction $I$ is an $\mathfrak{O}$-ideal, and $\kappa$ is the matrix of the linear transformation on $I$ of multiplication by $\alpha$.

Define an embedding $\iota: \mathfrak{O} \hookrightarrow \operatorname{Mat}_{2 \times 2}(\mathbb{Z})$ by

$$
\iota: x+y \alpha \mapsto x I+y \kappa .
$$

It follows that the determinant of $\iota(\beta)$ for $\beta \in \mathfrak{O}$ is the Galois norm $\mathcal{N}(\beta)$.

For $M \in \mathbb{N}$, define

$$
\tilde{\mathcal{A}}(M)=\left\{\beta \in \frac{\mathfrak{O}}{M \mathfrak{O}}: \mathcal{N}(\beta) \equiv 1 \quad \bmod M\right\},
$$

and

$$
\mathcal{A}(N)=\left\{\beta \in \tilde{\mathcal{A}}(2 N):\left\{\begin{array}{lll}
\beta \equiv 1 & \bmod 4 \mathfrak{O}, & (N \text { even }) \\
\beta \equiv 1 & \bmod 2 \mathfrak{O}, & (N \text { odd })
\end{array}\right\} .\right.
$$

Then $\iota(\mathcal{A}(N))$ consists of matrices belonging to $\Gamma(4,2 N)$ that commute with $\kappa$ and each other modulo $2 N$. It follows from (1) that the set of matrices $U_{N}(\iota(\beta))$ for $\beta \in \mathcal{A}(N)$ commute with each other and with $U_{N}(\kappa)$, and a joint basis of eigenvectors thereof is a Hecke basis.

A key step in the proof in [3] is an estimate for the quantity in (2) in the case that $\left\{\phi_{j}\right\}$ is a Hecke basis. 
Proposition $2([3])$. Let $\left\{\phi_{j}\right\}_{j=1}^{N}$ be a Hecke basis, and let $n \in \mathbb{Z}^{2} \backslash\{(0,0)\}$ and let $\nu=n_{1} v_{1}+n_{2} v_{2}$. Then

$$
\sum_{j=1}^{N}\left|\left\langle T_{N}(n) \phi_{j}, \phi_{j}\right\rangle\right|^{4} \leq \frac{N}{|\mathcal{A}(N)|^{4}} \mu(\nu),
$$

where $\mu(\nu)$ is the number of solutions to

$$
\nu\left(\beta_{1}-\beta_{2}+\beta_{3}-\beta_{4}\right) \equiv 0 \bmod N I,
$$

for $\beta_{1}, \ldots, \beta_{4} \in \mathcal{A}(N)$.

Thus quantum unique ergodicity for a Hecke basis is reduced to a counting problem for the number of solutions to $(\star)$ and of the size of $\mathcal{A}(N)$. The main result of [3] is the following:

Theorem 1 (Quantum unique ergodicity for Hecke bases, [3]). The following estimates hold: For $\nu=n_{1} v_{1}+n_{2} v_{2},\left(n_{1}, n_{2}\right) \neq(0,0)$,

$$
\mu(\nu) \ll|n|^{16} N^{2+\epsilon},
$$

and

$$
N^{1-\epsilon} \ll|\mathcal{A}(N)| \ll N^{1+\epsilon}
$$

It is easy to see that the estimates in theorem 1 taken together with proposition 2 leads to quantum unique ergodicity, using the criterion in proposition 1.

A later result [4] of Kurlberg and Rudnick establishes quantum unique ergodicity for any choice of basis, for a set of $N$ of density one in $\mathbb{N}$. The proof is based on the fact that quantum maps of the form discussed exhibit a periodicity. Define

$$
\operatorname{ord}(\kappa, N)=\min \left\{p \in \mathbb{N}: \kappa^{p} \equiv I \bmod N\right\} .
$$

Proposition $3([4])$. Let $\left\{\phi_{j}\right\}_{j=1}^{N}$ be any orthonormal basis, and let $n \in \mathbb{Z}^{2} \backslash$ $\{(0,0)\}$. Then

$$
\sum_{j=1}^{N}\left|\left\langle T_{N}(n) \phi_{j}, \phi_{j}\right\rangle\right|^{4} \ll|n|^{8+\epsilon} \frac{N(\log N)^{14}}{\operatorname{ord}(\kappa, N)^{2}} .
$$

The upshot of proposition 3 is that long periods imply quantum unique ergodicity for any basis, as a consequence of proposition 1.

Earlier, Degli Esposti, Graffi and Isola [2] had established (conditional on the Generalised Riemann Hypothesis) an infinite set of primes $N_{p}$ such that $\operatorname{ord}\left(\kappa, N_{p}\right) \gg N_{p}$, proving quantum unique ergodicity along that subsequence of $N_{p}$. Kurlberg and Rudnick prove the stronger result:

Theorem 2 (Quantum unique ergodicity for almost all $N,[4]$ ). For a subset of $N$ of density one in $\mathbb{N}$,

$$
\operatorname{ord}(\kappa, N) \gg N^{1 / 2} \exp \left((\log N)^{\delta}\right),
$$

for some $\delta>0$. 
We remark that it has been proved for certain subsequences of $N$ there exist choices of basis for which quantum unique ergodicity does not hold [5] (see also the contribution of Henrik Ueberschär), so theorems 1 and 2 cannot be improved in general.

\section{REFERENCES}

[1] J. H. Hannay and M. V. Berry, Quantization of linear maps-Fresnel diffraction by a periodic grating, Physica D 1 (1980) 267-290.

[2] M. Degli Esposti, S. Graffi and S. Isola, Classical limit of the quantized hyperbolic toral automorphisms, Commun. Math. Phys. 167 (1995) 471-507.

[3] P. Kurlberg and Z. Rudnick, Hecke theory and equidistribution for the quantization of linear maps of the torus, Duke Math. J. 103 (2000) 47-77.

[4] P. Kurlberg and Z. Rudnick, On quantum ergodicity for linear maps of the torus, Commun. Math. Phys. 222 (2001) 201-227.

[5] F. Faure, S. Nonnenmacher and S. De Bièvre, Scarred eigenstates for quantum cat maps of minimal periods, Commun. Math. Phys. 239 (2003) 449-492.

\section{Scars of periodic orbits}

KLAUS KRÖNCKE

In this talk, we discuss some physical predictions on the behaviour of eigenfunctions which will be underlined by some numerical results. We mainly consider the results obtained by [3] and [1]. Let $(M, g)$ be a Riemannian manifold and let $\Delta_{M}$ be the Laplace-Beltrami operator on $M$. Assume that $\psi_{n}$ is a normalized eigenfunction of $\Delta_{M}$ with eigenvalue $E_{n}$. It is shown that if $M$ is compact and without boundary, the $L_{p}$-norm of $\psi_{n}$ satisfies the estimate

$$
\left\|\psi_{n}\right\|_{p}<c E_{n}^{\delta(p, d)}
$$

where $d=\operatorname{dim}(M)$. In particular, in the two-dimensional case we know that $\left\|\psi_{n}\right\|_{\infty}<c E^{\frac{1}{4}}$. However, this estimate is believed being far away from sharp. If the system on $M$ is strongly chaotic,[2] conjectures that $\mid \psi_{n} \|$ behaves like a random superposition of plane waves which predicts a growth rate $\left\|\psi_{n}\right\|_{\infty} \propto \sqrt{\ln E_{n}}$. This is confirmed by numerical results on six chaotic two-dimensional billiards.

Furthermore, we discuss the rate of quantum ergodicity. An alternative formulation of quantum ergodicity which avoids the choice of a subsequence is given by

$$
\lim _{E \rightarrow \infty} \frac{1}{N(E)} \sum_{E_{n} \leq E}\left|\left\langle\psi_{n}, A \psi_{n}\right\rangle-\overline{\sigma(A)}\right|=0 .
$$

The rate of convergence is measured by the quantities

$$
S_{m}(E, A)=\frac{1}{N(E)} \sum_{E_{n} \leq E}\left|\left\langle\psi_{n}, A \psi_{n}\right\rangle-\overline{\sigma(A)}\right|^{m} \quad m \geq 1 .
$$



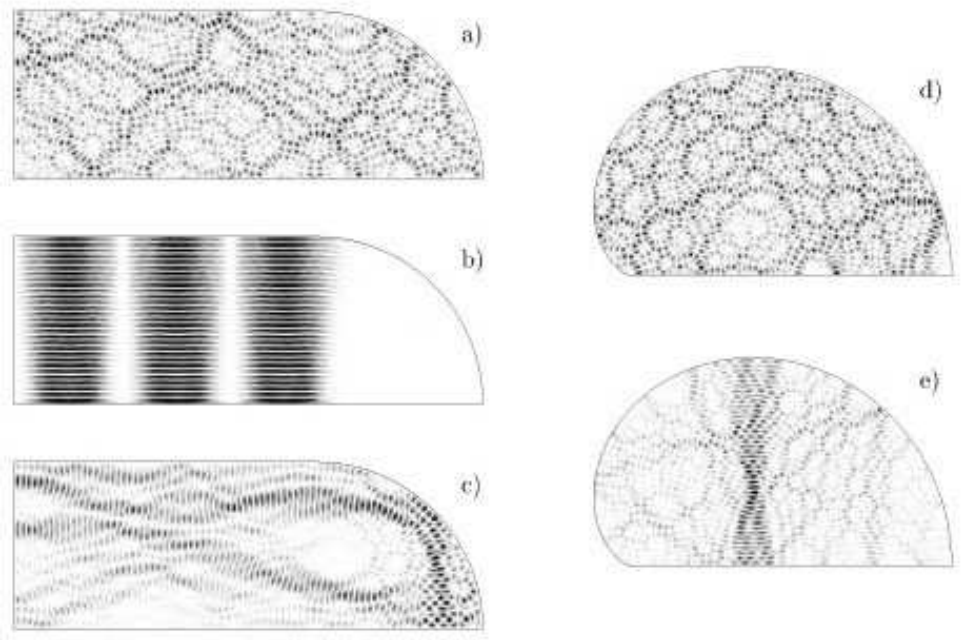

Figure 1. Density plots $\left|\psi_{n}(q)\right|^{2}$ for three different odd-odd eigenfunctions of the $a=1.8$ stadium billiard: a) $n=1992$, "generic" b) $n=1660$, bouncing ball mode, c)n=1771 localized eigenfunction. Right: Density plots of two eigenfunctions of the cardioid billiard with odd symmetry: d) $n=1816$, "generic", e) $n=1817$, localized along the $\overline{A B}$ orbit.

Let $\psi_{n^{\prime}}$ be a subsequence of eigenfunctions converging to the Liouville measure and let $\psi_{n^{\prime \prime}}$ be the complementary subsequence. Denote

$$
\begin{aligned}
& S_{m}^{\prime}(E, A)=\frac{1}{N^{\prime}(E)} \sum_{E_{n^{\prime}} \leq E}\left|\left\langle\psi_{n^{\prime}}, A \psi_{n^{\prime}}\right\rangle-\overline{\sigma(A)}\right|^{m} \quad m \geq 1 \\
& S_{m}^{\prime \prime}(E, A)=\frac{1}{N^{\prime \prime}(E)} \sum_{E_{n^{\prime \prime}} \leq E}\left|\left\langle\psi_{n^{\prime}}, A \psi_{n^{\prime}}\right\rangle-\overline{\sigma(A)}\right|^{m} \quad m \geq 1 .
\end{aligned}
$$

Clearly, we have

$$
S_{m}(E, A)=\frac{N^{\prime}(E)}{N(E)} S_{m}^{\prime}(E, A)+\frac{N^{\prime \prime}(E)}{N(E)} S^{\prime \prime}(E, A) .
$$

Assume for the quantum ergodic part of $S_{1}(E, A)$ a decay rate of $S_{1}^{\prime}(E, A)=$ $c E^{-\alpha}+o\left(E^{-\alpha}\right)$, and for the counting function of the not quantum-ergodic eigenfunctions $N^{\prime \prime}(E)=d E^{\beta}+o\left(E^{\beta}\right)$, where by quantum ergodicity $\alpha>0$ and $\beta<1$. It is conjectured that $\alpha=\frac{1}{4}$ which is supported by numerical results. It turns out that in this case, if we assume $\beta>\frac{3}{4}$, the non-ergodic eigenfunctions dominate the asymptotic behaviour of $S_{1}(E, A)$. More precisely, we then have $S_{1}(E, A)=$ $c E^{\beta-1}+o\left(E^{\beta-1}\right)$. In fact for every $\beta \in\left(\frac{1}{2}, 1\right)$, there exists a quantum system possessing a not quantum ergodic sequence of eigenfunctions whose counting function 
is asymptotically of order $E^{\beta}$. So without further assumptions than ergodicity, we have $S_{1}(E, A)=o(1)$.

At last, we discuss localization effects of not quantum-ergodic eigenfunctions. Mostly, one observes generic wave functions whose density looks irregular as examples a) and f) in the picture below shows. In billiards with two parallel walls, one observes the prescense of so-called bouncing ball modes which are localized around the so called bouncing-ball orbits, see example b). Other not quantumergodic eigenfunctions show localization effects in the neighbourhood of unstable periodic orbits, these are called "scars", see c) and e).

\section{REFERENCES}

[1] A. Bäcker, R. Schubert and P. Stifter, Rate of quantum ergodicity in Euclidean billiards, Phys. Rev. E 57 (1998), 5425-5447.

[2] M. V. Berry, Regular and irregular semiclassical wavefunctions, J. Phys. A 10 (1977), 20832091.

[3] R. Aurich, A. Bäcker, R. Schubert and M. Taglieber, Maximum norms of chaotic quantum eigenstates and random wave, Physica D 129 (1999), 1-14.

\section{A Counterexample to QUE \\ HENRIK UEBERSCHÄR}

Schnirelman's Theorem $[5,1,6]$ asserts that almost all eigenstates of a quantum system, whose classical counterpart is ergodic, equidistribute in the semiclassical limit. Possible exceptions are sparse subsequences of eigenstates which localise on periodic orbits in phase space. Quantum Unique Ergodicity (QUE) rules out the existence of such "scars". Whereas numerical evidence of scars has long been available [3], a rigorous construction was only recently provided by Faure, Nonnenmacher and de Bièvre [2].

This talk will illustrate the construction of half-scarred eigenstates for quantum cat maps of minimal quantum period. The quantum period of such quantum cat maps is of order $\log N$, where $N$ denotes the dimension of the Hilbert space, which leads to high degeneracies in the spectrum. They form an exception to the density one subsequence of quantum cat maps, with quantum period larger than $\sqrt{N}$, which was proven by Kurlberg and Rudnick [4] to equidistribute.

\section{BACKGROUND}

Let $A \in \mathrm{SL}(2, \mathbb{Z}),|\operatorname{Tr} A|>2$, be a hyperbolic automorphism of the torus $\mathbb{T}^{2}=\mathbb{R}^{2} / \mathbb{Z}^{2}$. Let $N=\frac{1}{2 \pi \hbar} \in \mathbb{N}$, and

$$
\theta= \begin{cases}(0,0), & N \text { even } \\ (\pi, \pi), & N \text { odd }\end{cases}
$$

and denote the associated Hilbert space

$$
\mathcal{H}_{N, \theta}=\left\{\psi \in \mathcal{S}^{\prime}(\mathbb{R}) \mid T_{1} \psi=T_{2} \psi=(-1)^{N} \psi\right\}
$$


where $T_{1} \psi(q)=\psi(q+1)$ and $T_{2} \psi(q)=e^{2 \pi \mathrm{i} q / N} \psi(q)$ are the quantum translation operators. Define the projection operator $P_{\theta}: \mathcal{S}(\mathbb{R}) \rightarrow \mathcal{H}_{N, \theta}$,

$$
P_{\theta}=\sum_{n \in \mathbb{Z}^{2}} e^{-\mathrm{i} n \cdot \theta} T_{1}^{n_{1}} T_{2}^{n_{2}}
$$

Denote by $U(A)=e^{-\mathrm{i} \hat{H} / \hbar}$ the quantum evolution operator on $L^{2}(\mathbb{R})$, where $\hat{H}$ is the quadratic Hamiltonian which generates the dynamics of $A$ acting on $\mathbb{R}^{2}$. Denote by $U_{N}(A)$ the quantum evolution operator on $\mathcal{H}_{N, \theta}$ which is defined by projection through the relation

$$
U_{N}(A) P_{\theta}=P_{\theta} U(A) .
$$

Let $w(x, k)=q k_{2}-p k_{1}, x=(q, p) \in \mathbb{T}^{2}$. The Weyl quantisation of a classical observable $f \in C^{\infty}\left(\mathbb{T}^{2}\right)$,

$$
f(x)=\sum_{k \in \mathbb{Z}^{2}} \hat{f}(k) e^{2 \pi \mathrm{i} w(x, k)}
$$

is given by

$$
O p(f)=\sum_{k \in \mathbb{Z}^{2}} \hat{f}_{k} T_{k / N}
$$

where $T_{y}=e^{\mathrm{i} w(\hat{x}, y) / \hbar}$ is the mixed quantum translation operator and $\hat{x}=(\hat{q}, \hat{p})$. We state the theorem in the simplest case.

Theorem $1([2])$. Let $A \in \mathrm{SL}(2, \mathbb{Z})$ be hyperbolic. There exists a subsequence $\left(N_{k}\right)_{k \in \mathbb{N}} \subset \mathbb{N}, \lim _{k \rightarrow \infty} N_{k}=\infty$, and a sequence of eigenfunctions $\left(\psi_{k}\right)_{k \in \mathbb{N}}$ of $U_{N_{k}}(A)$ in $\mathcal{H}_{N_{k}, \theta}$ such that for all $f \in C^{\infty}\left(\mathbb{T}^{2}\right)$

$$
\lim _{k \rightarrow \infty} \frac{\left\langle\psi_{k}, O p(f) \psi_{k}\right\rangle}{\left\|\psi_{k}\right\|_{2}^{2}}=\frac{1}{2} f(0)+\frac{1}{2} \int_{\mathbb{T}^{2}} f(x) d x .
$$

\section{Construction of the eigenstates}

We define the Gaussian wave packet $g_{0}=\frac{1}{2 \pi \hbar} e^{-q^{2} / 2 \hbar} \in L^{2}(\mathbb{R})$ with mean position $q=0$. We will construct the eigenstates $\psi_{k}$ as a superposition of time evolved Gaussian wave packets. To simplify computations we define the "squeezed" wave packet

where $Q \in \mathrm{SL}(2, \mathbb{Z})$ such that

$$
g_{s q}=U(Q) g_{0}
$$

$$
A=Q\left(\begin{array}{cc}
e^{\lambda} & 0 \\
0 & e^{-\lambda}
\end{array}\right) Q^{-1}
$$

and $\lambda$ denotes the Lyapunov exponent of $A$.

Minimal quantum periods. The quantum period of $U_{N}(A)$ is defined as the smallest non-negative integer such that

$$
U_{N}(A)^{P_{N}}=e^{\mathrm{i} \varphi_{N}} I d_{\mathcal{H}_{N, \theta}}
$$

for some $\varphi_{N} \in[0,2 \pi)$. The following Proposition is a crucial ingredient in the construction of the half-scarred eigenstates. 
Proposition 1 ([2]). Let $A \in \mathrm{SL}(2, \mathbb{Z}),|\operatorname{Tr} A|>2$. There exists a subsequence $\left(N_{k}\right)_{k \in \mathbb{N}} \subset \mathbb{N}, \lim _{k \rightarrow \infty} N_{k}=\infty$, such that

$$
P_{k}=2 T_{k}+O(1), \quad T_{k}=\frac{\log N_{k}}{\lambda},
$$

where $T_{k}$ is the Ehrenfest time of the system.

We construct the eigenstates as follows

$$
\psi_{k}=\sum_{t=-P_{k} / 2}^{P_{k} / 2-1} e^{-\mathrm{i} \theta_{k} t} U_{N_{k}}(A)^{t} P_{\theta} g_{s q}=\sum_{j=1}^{4} \psi_{k}^{j}
$$

where

$$
\theta_{k}=\frac{\varphi_{k}}{P_{k}} \quad \bmod \frac{2 \pi}{P_{k}}
$$

and

$$
\psi_{k}^{j}=\sum_{t=-P_{k} / 2+(j-1) P_{k} / 4}^{-P_{k} / 2+j P_{k} / 4-1} e^{-\mathrm{i} \theta_{k} t} U_{N_{k}}(A)^{t} P_{\theta} g_{s q} .
$$

One can easily check that

$$
\left(I d_{\mathcal{H}_{N_{k}, \theta}}-e^{-\mathrm{i} \theta_{k}} U_{N_{k}}(A)\right) \psi_{k}=0 .
$$

\section{SEmiclassical CONCENTRATion in Phase SPACE}

Let us introduce the Husimi function

$$
\mathcal{H}_{g_{s q}}(x)=\frac{1}{2 \pi \hbar}\left|\left\langle T_{x} g_{s q}, g_{s q}\right\rangle\right|^{2}, \quad x=(q, p) \in \mathbb{R}^{2}
$$

which measures the concentration of the wave packet $g_{s q}$ on the phase space $\mathbb{R}^{2}$. Similarly we define the Husimi function of the wave packet $P_{\theta} g_{s q} \in \mathcal{H}_{N, \theta}$ on the torus

$$
\mathcal{H}_{g_{s q}}^{\theta}(x)=N\left|\left\langle T_{x} g_{s q}, P_{\theta} g_{s q}\right\rangle\right|^{2}, \quad x=(q, p) \in \mathbb{T}^{2} .
$$

A simple calculation gives

$$
\mathcal{H}_{U(A)^{t} g_{s q}}(x)=\frac{1}{2 \pi \hbar \cosh (\lambda t)} \exp \left(-\frac{q^{\prime 2}}{\Delta q^{\prime 2}}-\frac{p^{\prime 2}}{\Delta p^{\prime 2}}\right),
$$

and

$$
\Delta q^{\prime 2}=\frac{2 \hbar}{1-\tanh (\lambda t)}, \quad p^{\prime 2}=e^{-2 \lambda t} \Delta q^{\prime 2}
$$

where $\left(q^{\prime}, p^{\prime}\right)=Q(q, p)$ is the unstable-stable frame of the classical dynamics.

So the evolved wave packet in the plane is concentrated in an ellipse which contracts along the stable axis and expands along the unstable axis of the classical dynamics as $t \rightarrow \infty$. The evolved wave packet on the torus is concentrated in a long thin ellipse which begins wrapping around the torus at time $t \approx T_{k} / 2$ and covers the torus at time $t \approx T_{k}$. At time $t=0$ the wave packet is concentrated in an ellipse of diameter $\sqrt{\hbar}$ and area $\hbar$. Note that mass is conserved under the time evolution so the wave packet starts out peaked and localised and flattens as it wraps around the torus. Time reversal symmetry implies that the behaviour of 
the evolved wave packet is analogous as $t \rightarrow-\infty$, where the expansion takes place along the stable axis and contraction along the unstable axis.

The states $\psi_{k}^{j}, j=1,2,3,4$ correspond to 4 different time regimes of the quantum dynamics during time $-T_{k} \leq t \leq T_{k}$. The states $\psi_{1}, \psi_{4}$ are spread out and contribute the volume measure of the torus in the semiclassical limit, whereas the states $\psi_{2}, \psi_{3}$ are localised and contribute the Dirac measure at $x=0$. We have the following Proposition.

Proposition $2([2])$. Let $f \in C^{\infty}\left(\mathbb{T}^{2}\right)$. We have

$$
\lim _{k \rightarrow \infty} \frac{\left\langle\psi_{k}^{j}, O p(f) \psi_{k}^{j}\right\rangle}{\left\|\psi_{k}^{j}\right\|_{2}^{2}}= \begin{cases}\int_{\mathbb{T}^{2}} f d \mu, & j=1,4 \\ f(0), & j=2,3\end{cases}
$$

The Theorem follows as a corollary of the Proposition.

\section{REFERENCES}

[1] Y. Colin de Verdière. Ergodicité et fonctions propres du laplacien. Comm. Math. Phys. 102 (1985) 497-502.

[2] F. Faure, S. Nonnenmacher, S. de Bièvre. Scarred eigenstates for quantum cat maps of minimal periods. Comm. Math. Phys. 239 (2003) 449-492.

[3] E. J. Heller. Bound-state eigenfunctions of classically chaotic hamiltonian systems: scars of periodic orbits. Phys. Rev. Lett. 53 (1984) 1515-18.

[4] P. Kurlberg, Z. Rudnick. On quantum ergodicity for linear maps of the torus. Comm. Math. Phys. 222 (2001) 201-27.

[5] A. Schnirelman. Ergodic properties of eigenfunctions. Uspekhi Math. Nauk. 29 (1974) 181-2

[6] S. Zelditch. Uniform distribution of eigenfunctions on a compact hyperbolic surface. Duke Math. J. 55 (1987) 919-41.

\section{Hassell's Proof of Quantum Non-Unique Ergodicity for the Bunimovich Stadium \\ DAVID DAMANIK}

We consider the Bunimovich stadium $S_{t}=R_{t} \cup W_{t}$, where $R_{t}=[-t \pi / 2, t \pi / 2] \times$ $[-\pi / 2, \pi / 2]$ denotes the rectangle and $W_{t}$ denotes the union of the two wings, that is, the two half-disks of radius $\pi / 2$ and centers $( \pm t \pi / 2,0)$.

We denote the Dirichlet Laplacian on $S_{t}$ by $\Delta_{t}$ (chosen to be a positive operator, i.e., acting as $\left.-\partial_{x}^{2}-\partial_{y}^{2}\right)$. It has compact resolvent and hence purely discrete spectrum. We denote by $E_{j}(t)$ (resp., $\left.u_{j}(t)\right)$ the $j$-th eigenvalue (resp., $L^{2}\left(S_{t}\right)$ normalized eigenfunction) of $\Delta_{t}$ (counted with multiplicity).

The unit co-sphere bundle $S^{*} S_{t}$ serves as the phase space of the billiard dynamics. To compare the classical mechanical system with a quantum mechanical system, one uses a quantization rule, which maps observables, or symbols, to pseudodifferential operators. That is, given $a \in C^{\infty}\left(S^{*} S_{t}\right)$, there is an associated semi-classical pseudodifferential operator $A_{h}$ on $L^{2}\left(S_{t}\right)$. Given such an operator $A_{h}$, its principal symbol is denoted by $\sigma_{0}(A)$. 
We say that $S_{t}$ is quantum ergodic if there is a set $J \subseteq \mathbb{Z}_{+}$of density one such that for each semi-classical pseudodifferential operator $A_{h}$ of order zero, whose symbol is compactly supported in the interior, we have

$$
\lim _{J \ni j \rightarrow \infty}\left\langle A_{h_{j}} u_{j}(t), u_{j}(t)\right\rangle=\frac{1}{\mu\left(S^{*} S_{t}\right)} \int_{S^{*} S_{t}} \sigma_{0}(A) d \mu,
$$

where $d \mu$ is the Liouville measure on $S^{*} S_{t}$. Here, $h_{j}=E_{j}(t)^{-1 / 2}$ is the length scale corresponding to $u_{j}(t)$. If $J$ can be chosen to be all of $\mathbb{Z}_{+}$, we say that $S_{t}$ exhibits quantum unique ergodicity.

The main result from [3] is the following:

Theorem 1. For Lebesgue almost every $t \in[1,2]$, quantum unique ergodicity fails for $S_{t}$.

The heuristic reason for this result is the existence of bouncing ball orbits for the billiard. That is, there are periodic vertical trajectories that never visit the wings of the stadium. Numerics have shown that some eigenfunctions concentrate on such bouncing ball orbits. To prove Theorem 1, one has to show that this behavior persists in the high-energy limit. To do this, one first exhibits so-called quasimodes that do indeed concentrate on bouncing ball orbits. Then one expands these states in eigenfunctions. One has to show that infinitely often, only a uniformly bounded number of eigenfunctions suffices to exhaust a significant part of this expansion. Thus, picking the one with maximal expansion coefficient, one obtains an infinite sequence of eigenfunctions which has uniformly significant weight near bouncing ball orbits. This shows that quantum unique ergodicity fails since the bouncing ball orbits have zero weight with respect to Liouville measure.

We first introduce the quasi-modes. Consider a function $v_{n} \in D\left(\Delta_{t}\right)$ given by

$$
v_{n}(x, y)= \begin{cases}\chi(x) \sin n y & n \text { even, } \\ \chi(x) \cos n y & n \text { odd }\end{cases}
$$

where $\chi$ is smooth, supported in $[-\pi / 4, \pi / 4]$, and such that $\left\|v_{n}\right\|_{L^{2}\left(S_{t}\right)}=1$. The first result, which follows quickly from the spectral theorem, shows that the energy of $v_{n}$ is essentially localized around $n^{2}$.

Lemma 1. We have $\left\|\left(\Delta_{t}-n^{2}\right) v_{n}\right\|_{L^{2}\left(S_{t}\right)} \leq K$, uniformly in $n \in \mathbb{Z}_{+}$and $t \in[1,2]$, and therefore $\left\|\chi_{\left[n^{2}-2 K, n^{2}+2 K\right]}\left(\Delta_{t}\right) v_{n}\right\|_{L^{2}\left(S_{t}\right)}^{2} \geq \frac{3}{4}$.

Since the spectrum of the Laplacian is discrete, only a finite number of eigenvalues lies in the interval $\left[n^{2}-2 K, n^{2}+2 K\right]$. This finite number, however, may be $n$-dependent and in particular unbounded. Since we need uniformity infinitely often, the following condition is natural.

Definition 1. We say that condition (HOZ) holds for $t \in[1,2]$ if there exists a constant $M$ and a sequence $n_{j} \rightarrow \infty$ of integers such that for each $j$, $\operatorname{dim} \chi_{\left[n_{j}^{2}-2 K, n_{j}^{2}+2 K\right]}\left(\Delta_{t}\right) \leq M$. 
Lemma 2. Suppose condition (HOZ) holds for $t \in[1,2]$ with some constant $M$. Then, there exists a quantum limit having mass at least $\frac{3}{4 M}$ on the bouncing ball trajectories. In particular, quantum unique ergodicity fails for $S_{t}$.

This reduces the proof of Theorem 1 to showing that condition (HOZ) holds for Lebesgue almost every $t \in[1,2]$. To accomplish this, one studies how the eigenvalues vary with $t$. The idea here is that, while unusual accumulation of eigenvalues may be possible for some $t$, this quickly dissolves once $t$ is varied. To implement this, the following formula is crucial:

Lemma 3 (Hadamard Variational Formula). With the normal variation $\rho_{t}$ of the boundary $\partial S_{t}$ and $\psi_{j}(s)=E_{j}(t)^{-1 / 2} d_{n} u_{j}(t)(s)$, we have

$$
E_{j}(t)^{-1} \frac{d}{d t} E_{j}(t)=-\int_{\partial S_{t}} \rho_{t}(s) \psi_{j}(s)^{2} d s .
$$

Theorem 1 now follows from the following explicit implementation of the heuristic idea:

Proposition 1. For every $\varepsilon>0$, there exists a subset $B_{\varepsilon}$ of $[1,2]$ of Lebesgue measure at least $1-4 \varepsilon$, and $M(\varepsilon)<\infty$ such that for every $t \in B_{\varepsilon}$, condition (HOZ) holds with constant $M(\varepsilon)$.

Proof. Define

$$
\begin{aligned}
f_{j}(t) & =\int_{\partial S_{t}} \rho_{t}(s) \psi_{j}(s)^{2} d s, \\
Z_{1} & =\left\{t \in[1,2]: \liminf _{j \rightarrow \infty} f_{j}(t)=0\right\}, \\
Z_{2} & =\left\{t \in[1,2]: \liminf _{j \rightarrow \infty} f_{j}(t)>0\right\} .
\end{aligned}
$$

First, consider $t \in Z_{1}$. It follows from earlier work by Burq-Gérard and GérardLeichtnam $[1,2]$ that for each such $t$ there exists a quantum limit that is fully supported on interior rays that do not meet the wings of the stadium. The only such trajectories are the bouncing ball trajectories. Therefore, every $t \in Z_{1}$ may be added to the set $B_{\varepsilon}$ for every $\varepsilon>0$.

Now we consider $t$ 's in the set $Z_{2}$. Let $\varepsilon>0$ be given. The main steps are the following:

Step 1. There are $G \subseteq Z_{2}, N \in \mathbb{Z}_{+}$, and $c$ such that

$$
|G|=\left|Z_{2}\right|-2 \varepsilon
$$

and

$$
t \in G, j \geq N \quad \Rightarrow \quad f_{j}(t) \geq c .
$$

Step 2. There is a constant $\gamma>0$ such that for every $a>0$, we can find for $n \in \mathbb{Z}_{+}$large enough, a set $A_{n} \subset G$ of measure $\left|A_{n}\right| \geq\left|Z_{2}\right|-3 \varepsilon$ such that for 
every $t \in A_{n}$, we have

$$
N_{t}\left(n^{2}+a\right)-N_{t}\left(n^{2}-a\right) \leq \frac{12 a}{c \gamma \varepsilon} .
$$

Step 3. With $B_{k}=\left\{t \in Z_{2}: t \in A_{n}\right.$ for at least $k$ distinct values of $\left.n\right\}$, we have

$$
\left|\bigcap_{k} B_{k}\right| \geq\left|Z_{2}\right|-4 \varepsilon
$$

One can then finish the proof as follows. For $t \in \bigcap_{k>n_{0}} B_{k}$, which is a set of measure $\geq\left|Z_{2}\right|-4 \varepsilon$ by Step 3, there is a (t-dependent) sequence $n_{j} \rightarrow \infty$ such that $N_{t}\left(n_{j}^{2}+a\right)-N_{t}\left(n_{j}^{2}-a\right) \leq \frac{24 a}{c \gamma \varepsilon}$ for each $j$ by choice of $t$ and Step 2. In other words, condition (HOZ) holds for $t$ and hence Lemma 2 yields the assertion.

\section{REFERENCES}

[1] N. Burq and P. Gérard, Condition nécessaire et suffisante pour la contrôlabilité exacte des ondes, C. R. Acad. Sci. Paris Sér. I Math. 325 (1997), 749-752.

[2] P. Gérard and É. Leichtnam, Ergodic properties of eigenfunctions for the Dirichlet problem, Duke Math. J. 71 (1993), 559-607.

[3] A. Hassell, Ergodic billiards that are not quantum unique ergodic. With an appendix by the author and Luc Hillairet, Ann. of Math. 171 (2010), 605-619.

\section{Entropy bounds for semiclassical measures SEMYON Dyatlov}

According to the quantum unique ergodicity (QUE) conjecture of Rudnik and Sarnak, eigenmodes of manifolds of negative sectional curvature should be equidistributed in the high energy limit. If $u_{j}$ are the eigenfunctions of Laplace's operator on a compact manifold $M^{d}$, we call a measure $\mu$ on the cotangent bundle $T^{*} M$ a semiclassical measure if it can be expressed as the limit of a subsequence $\left(u_{j_{k}}\right)$ in the sense that $\left\langle\mathrm{Op}_{h}(a) u_{j_{k}}, u_{j_{k}}\right\rangle \rightarrow \int a d \mu$ for a semiclassical quantization $\mathrm{Op}_{h}(a): L^{2}(M) \rightarrow L^{2}(M)$ of any classical observable $a \in C_{0}^{\infty}\left(T^{*} M\right)$. The quantum ergodicity theorem of Shnirelman, Colin de Verdière, and Zelditch states in particular that on a manifold of negative curvature, a density one subsequence of $u_{j}$ converges to the Liouville measure $\mu_{0}$; the QUE conjecture states that $\mu_{0}$ is the only semiclassical measure.

This lecture presents the result [Ana] which can be seen as partial progress towards the QUE conjecture. The result can be summarized as follows: for manifolds with Anosov geodesic flow, few components of the ergodic decomposition of any semiclassical measure can have low entropy. The Anosov property, true for manifolds of negative curvature, means that the phase space can be decomposed into the direction of the flow, the stable directions, and the unstable directions, where the flow shrinks exponentially along the stable directions for large positive times, and the unstable directions for large negative times. How low the entropy 
is allowed to be depends on the unstable Jacobian of the flow. This in particular shows that no semiclassical measure can have low entropy; for example, no semiclassical measure can be supported on a periodic trajectory.

The fundamental idea behind the statement is that each quantum object has to be concentrated on a set of measure at least $h^{d}$, because of the uncertainty principle. The eigenfunctions are quantum objects invariant under the Schrödinger propagator, quantizing the geodesic flow. Since this flow is Anosov, if we propagate the eigenfunction for time $t$ (arriving to the same eigenfunction modulo a constant factor), it spreads out along the unstable directions and shrinks along the stable directions. However, if the measure is concentrated on a set of small entropy, the amount by which the eigenfunctions contract along the stable directions overpowers the complexity of the flow, expressed by the entropy, and we arrive to a contradiction.

However, one can only use the fact that $u_{j}$ converge to $\mu$ on fixed sets, which corresponds to considering symbolic dynamics for finite time, while the hyperbolicity estimate gives a lower bound on the number of segments needed to cover the support of the measure for symbolic dynamics at long logarithmic time in $h$. To overcome this difficulty, we first make a combinatorial argument to estimate the measure of a certain neighborhood $W$ of the support of the measure in terms of the measure of the set $\Sigma_{N_{0}}(W)$, consisting of symbolic strings which have many substrings lying in $W$; here $N_{0}$ is a small logarithmic time. We need $N_{0}$ to be below the Ehrenfest time because the argument uses the fact that the quantum measures are almost positive, which requires that the associated operators be pseudodifferential. We then conclude by using a submultiplicativity argument to relate the measure of $\Sigma_{N_{0}}(W)$ to certain information at a large logarithmic time, which will contradict the hyperbolicity estimate.

\section{REFERENCES}

[Ana] Nalini Anantharaman, Entropy and the localization of eigenfunctions, Ann. Math. 168 (2008), 435-475 


\section{Participants}

Prof. Dr. Bernd Ammann

Fakultät für Mathematik

Universität Regensburg

93040 Regensburg

Prof. Dr. Ulrich Bunke

Fakultät für Mathematik

Universität Regensburg

93040 Regensburg

Prof. Dr. Brice Camus

Fakultät für Mathematik

Ruhr Universität Bochum

Universitätsstr. 150

44780 Bochum

Prof. Dr. David Damanik

Dept. of Mathematical Sciences

Rice University

P. O. Box 1892

Houston , TX 77251

USA

\section{Dzmitry Dudko}

School of Engineering and Science Jacobs University Bremen

Postfach 750561

28725 Bremen

\section{Semyon Dyatlov}

Department of Mathematics

University of California, Berkeley

Evans Hall

Berkeley , CA 94720-3840

USA

\section{Dr. Andrej V. Ershov}

Mathematisches Institut

Georg-August-Universität Göttingen

Bunsenstr. 3-5

37073 Göttingen
Prof. Dr. Gerd Faltings

Max-Planck-Institut für Mathematik

Vivatsgasse 7

53111 Bonn

\section{Karsten Fritzsch}

Institut fuer Mathematik

Carl v. Ossietzky-Universität Oldenburg

Fakultät V: Mathematik \& Naturwissensch.

26111 Oldenburg

\section{Peter Gmeiner}

Department Mathematik

Universität Erlangen-Nürnberg

Bismarckstr. 1 1/2

91054 Erlangen

\section{Prof. Dr. Sebastian Goette}

Mathematisches Institut

Universität Freiburg

Eckerstr. 1

79104 Freiburg

\section{Prof. Dr. Colin Guillarmou}

Dept. de Mathematiques et Applications

Ecole Normale Superieure

45, rue d'Ulm

F-75230 Paris Cedex 05

\section{Prof. Dr. Roland Gunesch}

Department Mathematik

Universität Hamburg

Bundesstr. 55

20146 Hamburg

\section{Ilya Khayutin}

Institute of Mathematics

The Hebrew University

Givat-Ram

91904 Jerusalem

ISRAEL 
Klaus Kröncke

Fachbereich Mathematik

Universität Potsdam

Am Neuen Palais 10

14469 Potsdam

\section{Haruya Mizutani}

Department of Mathematics

Kyoto University

Kitashirakawa, Sakyo-ku

Kyoto 606-8502

JAPAN

Dr. Carolina Neira Jimenez

Fakultät für Mathematik

Universität Regensburg

Universitätsstr. 31

93053 Regensburg

Dr. Stephane Nonnenmacher

Institut de Physique Theorique

CEA Saclay

F-91191 Gif-sur-Yvette Cedex

Prof. Dr. Martin Olbrich

Universite du Luxembourg

Unite de Recherche en Mathematiques

6, Rue Richard Coudenhove-Kalergi

L-1359 Luxembourg

Prof. Dr. Anna Maria Paolucci

Dipartimento di Matematica

Politecnico di Torino

Corso Duca degli Abruzzi, 24

I-10129 Torino

\section{Aprameyan Parthasarathy}

Fachbereich Mathematik

Universität Marburg

Hans-Meerwein-Str.

35043 Marburg
Dr. Anke Pohl

Departement Mathematik

ETH-Zentrum

Rämistr. 101

CH-8092 Zürich

Dr. Nicole Raulf

Universite de Lille 1

U.F.R. de Mathematiques Pures

et Appliquees

F-59655 Villeneuve d' Ascq. Cedex

Dr. Lior Rosenzweig

Einstein Institute of Mathematics

The Hebrew University

Givat Ram

91904 Jerusalem

ISRAEL

Dr. Julie Rowlett

Max-Planck-Institut für Mathematik

Vivatsgasse 7

53111 Bonn

Nils Schippkus

Fakultät für Mathematik

Ruhr-Universität Bochum

Universitätsstr. 150

44801 Bochum

Prof. Dr. Dierk Schleicher

School of Engineering and Science

Jacobs University Bremen

Postfach 750561

28725 Bremen

Dr. Roman Schubert

Department of Mathematics

University of Bristol

University Walk

GB-Bristol BS8 1TW 


\section{Dr. Holger Then}

School of Mathematics

University of Bristol

Howard House

Queens Avenue

GB-Bristol BS8 1SN

\section{Dr. Henrik Ueberschär}

School of Mathematical Sciences

Tel Aviv University

Ramat Aviv

Tel Aviv 69978

ISRAEL

Dr. Pankaj H. Vishe

Department of Mathematics

Royal Institute of Technology

Lindstedtsvägen 25

S-100 44 Stockholm

\section{Dr. Brian Winn}

Dept. of Mathematical Sciences

Loughborough University

Loughborough

GB-Leicestershire LE11 3TU

\section{Prof. Dr. Ingo Witt}

Mathematisches Institut

Georg-August-Universität Göttingen

Bunsenstr. 3-5

37073 Göttingen 
